\title{
Extended balancing of continuous LTI systems: a structure-preserving approach
}

\author{
Pablo Borja ${ }^{1}$, Jacquelien M.A. Scherpen ${ }^{1}$, and Kenji Fujimoto ${ }^{2}$ \\ ${ }^{1}$ Jan C. Willems Center for Systems and Control, ENTEG, FSE, \\ University of Groningen. The Netherlands. \\ Email:1.p.borja.rosales [j.m.a.scherpen]@rug.nl \\ ${ }^{2}$ Dept of Aeronautics and Astronautics, \\ Kyoto University, Japan. \\ Email:fujimoto@kuaero.kyoto-u.ac.jp.
}

\begin{abstract}
In this paper, we treat extended balancing for continuous-time linear time-invariant systems, and we address the problem of structurepreserving model reduction of the subclass of port-Hamiltonian systems. We establish sufficient conditions to ensure that the reduced-order model preserves a port-Hamiltonian structure. Moreover, we show that the use of extended Gramians can be exploited to get a small error bound and, possibly, to preserve a physical interpretation for the reduced-order model.
\end{abstract}

Keywords: port-Hamiltonian systems, model reduction, extended Gramians, error bound.

\section{Introduction}

Balancing is a tool that is often used for model reduction purposes, giving rise to the balanced truncation methodology. This approach relies on realization theory, observability and controllability Gramians and is directly related to the concept of Hankel operator of a system. Moreover, since its introduction in the seminal work of Moore [14, balancing for stable linear systems has been extensively studied, in particular, a thorough exposition of this topic can be found in [1, while in [18, a brief tutorial is presented, which provides the basis for extending the results to nonlinear systems 9 .

Balanced truncation, based on the use of standard observability and controllability Gramians, preserves some appealing properties of the original system, e.g., asymptotic stability, observability and controllability. Furthermore, it is possible to establish an error bound, which is given in terms of the so-called Hankel singular values [10 corresponding to the truncated states. Nevertheless, in this standard formulation of balanced truncation some properties of the original system, like passivity or particular structures, are not necessarily preserved. Another possible drawback of this approach takes place when the Hankel singular values are large, which gives origin to a large error bound. Accordingly, with the aim of dealing with the latter issue, the use of the so-called gener- 
alized Gramians for model reduction purposes was introduced in 11. Where the generalized observability and controllability Gramians are solutions to the respective Lyapunov inequalities, this differs from the definition of the standard Gramians which are given by the solutions of the Lyapunov equalities. Furthermore, it has been proven that it is possible to preserve some important properties while using balanced truncation based on the use of generalized Gramians. Moreover, since the solutions of the before mentioned Lyapunov inequalities are not unique, generalized Gramians can be used to obtain a smaller error bound [7, and in some cases, to preserve some interesting structures [3].

A further extension of balanced truncation can be formulated by using the concept of extended Gramians, which, for the discrete-time versions were introduced in 17]; and a preliminary continuous-time counter part of these results was recently reported in 19 . The discrete-time and continuous-time methods are rather different, except from the fact that the disspativity theory plays a fundamental role in both to establish the error bound. In this approach, referred as extended balancing, the Gramians are solutions to specific linear matrix inequalities (LMIs) and, in contrast to other balancing methods, the error bound is obtained by using dissipativity arguments 21 and not through a transfer function approach. Furthermore, this balancing method provides more degrees of freedom to impose certain structure to the reduced order model, and can be potentially useful to improve the error bound.

In this work, we focus on the extended balanced truncation of continuous-time linear time-invariant (CTLTI) systems, where we are interested in the versatility of this methodology to preserve particular structures. Notably, we pay special attention to CTLTI port-Hamiltonian (PH) systems which are suitable to represent several physical systems, e.g., RLC circuits and mechanical systems; and are endowed with interesting properties, such as passivity. Therefore, the objective of this work is not only to reduce the order of the original system, but also to preserve its $\mathrm{PH}$ structure. Towards this end, we first study extended balanced truncation for CTLTI systems, and then we focus on its application to CTLTI PH with structure preservation purposes. The main contributions of this paper are given as follows:

- We recall the results from 19, and provide proofs for the error bound computation which turn out to be rather different than in the dicretetime case.

- We identify a family of generalized Gramians that are suitable for balanced truncation of CTLTI PH systems with PH structure preservation. To the best of our knowledge, the characterization of these solutions to the Lyapunov inequalities is new.

- The use of extended balancing as a tool to design a small error bound. Moreover, we show with an illustrative example that this approach can be used to preserve more particular structures, like RLC circuits structure, and a physical interpretation for the reduced order model.

The remainder of the paper is organized in the following manner: we provide the basic background in Section 2, while the fundamental notion of extended Gramians and the computation of the error bound are presented in Section 3. In Section 4, we introduce the generalized and extended balancing of PH systems with structure preservation. We present two illustrative examples in Section 5 , where the use of extended Gramians in the second example allow us to preserve 
an even more particular structure than the $\mathrm{PH}$ one, that is, the reduced order system is physically interpretable as an RLC circuit again. Finally, in Section 66 we wrap-up this note with some concluding remarks.

Notation: We assume that all the matrices have exclusively real entries. Consider a symmetric matrix $A \in \mathbb{R}^{n \times n}$, then $A$ is positive semi-definite if $x^{\top} A x \geq$ $0, \forall x \in \mathbb{R}^{n}$. Moreover, $A$ is positive definite if $x^{\top} A x>0, \forall x \in \mathbb{R}^{n} \backslash\{0\}$. The identity matrix is denoted as $I$, when necessary a subscript is added to indicate the dimension of the matrix. The symbol $\mathbf{0}_{q \times p}$ denotes a matrix of dimensions $q \times p$ whose entries are zeroes The set of positive real numbers is expressed as $\mathbb{R}_{>0}$, while, the set of nonnegative real numbers is denoted by $\mathbb{R}_{\geq 0}$. In the sequel, the symbol $\Lambda$ is reserved for diagonal matrices with positive entries, that is, the square matrix $\Lambda \in \mathbb{R}^{n \times n}$ is given by $\Lambda=\operatorname{diag}\left\{\sigma_{1}, \cdots, \sigma_{n}\right\}$, where $\sigma_{i} \in \mathbb{R}_{>0}$, for $i=1, \cdots n$. The symbol $U$ is reserved to orthogonal matrices, that is, $U U^{\top}=I$. Consider the vector $x \in \mathbb{R}^{n}$, then $|x|$ denotes the Euclidean norm of $x$, that is, $|x|=\sqrt{x^{\top} x}$. Let $e \in \mathbb{R}^{n}$ be a signal, then $\|e\|_{2}$ denotes the $\mathcal{L}_{2}$ norm of $e$, namely, $\|e\|_{2}=\left(\int_{0}^{\infty}|e(t)|^{2} d t\right)^{\frac{1}{2}}$.

\section{Preliminaries}

Consider a continuous-time linear time-invariant (CTLTI) system described as

$$
\Sigma:\left\{\begin{array}{l}
\dot{x}=A x+B u \\
y=C x
\end{array}\right.
$$

where $x \in \mathbb{R}^{n}$ is the state vector, for $m \leq n, u \in \mathbb{R}^{m}$ is the input vector and $y \in \mathbb{R}^{q}$ denotes the output vector. Accordingly, $A \in \mathbb{R}^{n \times n}, B \in \mathbb{R}^{n \times m}$ and $C \in \mathbb{R}^{q \times n}$. Assume that the system (10) is asymptotically stable, thus, the socalled generalized observability Gramians $Q \in \mathbb{R}^{n \times n}$ are positive semi-definite solutions to the following Lyapunov inequality

$$
Q A+A^{\top} Q+C^{\top} C \leq 0 .
$$

Analogously, the generalized controllability Gramians $\breve{P} \in \mathbb{R}^{n \times n}$ are given by positive semi-definite solutions to

$$
A \breve{P}+\breve{P} A^{\top}+B B^{\top} \leq 0 .
$$

In particular, when (2) and (3) are equalities, the matrices $Q$ and $\breve{P}$ are known as the standard observability and controllability Gramian, respectively. For further details, we refer the reader to 1 .

\subsection{Generalized balanced truncation for LTI}

A CTLTI system is said to be generalized balanced if

$$
Q=\breve{P}=\Lambda_{Q P},
$$

where $\Lambda_{Q P}>0$ is a diagonal matrix, see the Notation section. Accordingly, balancing for LTI systems, 14, relies on obtaining an invertible state transformation

$$
\bar{x}=W_{g}^{-1} x
$$


such that

$$
W_{g}^{-1} \breve{P} Q W_{g}=\Lambda_{Q P}^{2},
$$

where we assume that the elements of $\Lambda_{Q P}=\operatorname{diag}\left\{\sigma_{1}, \cdots, \sigma_{n}\right\}$ are ordered from largest to smallest, that is, $\sigma_{i}>\sigma_{i+1}$, for $i=1, \cdots, n-1$. Model reduction based on balancing is carried out by truncating the states corresponding to the small elements of $\Lambda_{Q P}$, i.e., if $\sigma_{i}>>\sigma_{i+1}$, then we set

$$
\bar{x}_{i+1}=\cdots=\bar{x}_{n}=0 .
$$

The error bound is given by the sum of the truncated singular values [10, i.e.,

$$
\|\Sigma-\widehat{\Sigma}\|_{\infty} \leq 2 \sum_{j=i+1}^{n} \sigma_{j},
$$

where $\widehat{\Sigma}$ corresponds to the realization of the reduced order system. For a more elaborated exposition of balancing and the corresponding reduced order model properties, we refer the reader to 22. At this point, we highlight that the error bound obtained through generalized balanced truncation is lower than the one obtained with the use of standard Gramians, for further details see [11].

\section{Extended balanced truncation}

The generalized balanced truncation approach can be extended by considering the so-called extended Gramians instead of the generalized ones. This extension has two main advantages: on one hand, the error bound can be reduced as has been shown in [16] for the discrete-time case. On the other hand, the use of extended Gramians provides extra degrees of freedom which can be exploited to impose a certain structure on the reduced order system.

In this section we revisit and significantly improve the concept of extended balanced truncation for the continuous-time case, which was first introduced in [19]. Towards this end, we introduce the following assumption which is necessary to establish the concept of extended Gramians.

Assumption 1. There exist strictly positive solutions, $Q, \breve{P}$, to inequalities (22) and (3).

We stress the fact that if the system (1) is controllable and observable, then Assumption 1 holds. Nonetheless, this latter condition is sufficient but not necessary, thus, might be conservative. Moreover, if Assumption 1 is satisfied, then we can define

$$
P:=\breve{P}^{-1} .
$$

Note that $P$ is a positive definite matrix.

Before introducing the concept of extended Gramians we define the following matrices

$$
\begin{aligned}
A_{o} & :=\alpha I_{n}+A, \\
A_{c} & :=\beta I_{n}+A, \\
X_{o} & :=-Q A-A^{\top} Q-C^{\top} C, \\
X_{c} & :=-P A-A^{\top} P-P B B^{\top} P, \\
Y_{c} & :=-P+\left(A_{c}^{\top}+P B B^{\top}\right) T,
\end{aligned}
$$


where $P$ is defined in (9), $\alpha \in \mathbb{R}_{>0}$, and $\beta \in \mathbb{R}_{\geq 0}$. Furthermore, from (2) and (3), it follows that $X_{o} \geq 0, X_{c} \geq 0$.

The definition of extended Gramians is the starting point of the theory contained in the following sections of this paper. These concepts were introduced for CTLTI systems without proofs in [19. Below we present the, slightly altered, results and their corresponding proof.

Extended Gramians. Consider the following two LMIs.

$$
\left[\begin{array}{cc}
X_{o} & Q-A_{o}^{\top} S \\
Q-S^{\top} A_{o} & S+S^{\top}
\end{array}\right] \geq 0
$$

and

$$
\left[\begin{array}{ccc}
-P A-A^{\top} P & -P+A_{c}^{\top} T & -2 P B \\
-P+T^{\top} A_{c} & T+T^{\top} & 2 T^{\top} B \\
-2 B^{\top} P & 2 B^{\top} T & 4 I_{m}
\end{array}\right] \geq 0
$$

with $T, S \in \mathbb{R}^{n \times n}$. We call (10) and (11) the extended observability and controllability LMIs with extended observability Gramian $(Q, S, \alpha)$ and extended inverse controllability Gramian $(P, T, \beta)$, respectively.

Now we are in position to formulate the relation between the generalized observability Gramian and the extended observability Gramian.

Theorem 1. (observability Gramians)

The inequality (2) has a solution $Q>0$ if and only if the LMI (10) admits a solution $(Q, S, \alpha)$ with $Q>0,\left(S+S^{\top}\right) \geq 0$, and $\alpha$ large enough. Moreover, if $X_{o}>0$, then there exists an $\alpha$ large enough, and $S=S^{\top}>0$ such that the LMI (10) holds.

Proof. Only if. Assume that (10) has a solution $(Q, S, \alpha)$, then multiplying (10) by $\left[\begin{array}{ll}I_{n} & \mathbf{0}_{n \times n}\end{array}\right]$ from the left and by $\left[\begin{array}{ll}I_{n} & \mathbf{0}_{n \times n}\end{array}\right]^{\top}$ from the right, it follows that (2) admits a solution $Q>0$.

If. Assume there exists $Q>0$ solving (21). Select $S=A_{o}^{-\top} Q$, with $-\alpha$ not an eigenvalue of $A$. Then, the off-diagonal blocks of (10) are zero. Furthermore,

$$
S+S^{\top}=A_{o}^{-\top} Q+Q A_{o}^{-1} .
$$

Accordingly, we have the following equivalence

$$
\begin{aligned}
0 \leq S+S^{\top} \Longleftrightarrow & \\
0 \leq A_{o}^{\top}\left(S+S^{\top}\right) A_{o} & =A_{o}^{\top} Q+Q A_{o} \\
& =2 \alpha Q-C^{\top} C-X_{o} .
\end{aligned}
$$

Note that, since $X_{o}$ does not depend on $\alpha$, the inequality (13) holds for $\alpha$ large enough. Hence, there exist $Q>0$ and $\alpha>0$ such that LMI (10) holds.

Symmetric $S$. Assume that $Q>0$ and $X_{o}>0$. Consider a symmetric matrix $\Gamma_{o} \in \mathbb{R}^{n \times n}$ verifying

$$
\alpha Q+\Gamma_{o}>0
$$

Select

$$
S=Q\left(\alpha Q+\Gamma_{o}\right)^{-1} Q .
$$


Hence, $S=S^{\top}>0$. Now, multiply (10) by $\operatorname{diag}\left\{I_{n}, Q S^{-1}\right\}$ from the left and by $\operatorname{diag}\left\{I_{n}, S^{-1} Q\right\}$ from the right, yielding

$$
\begin{aligned}
& {\left[\begin{array}{cc}
X_{o} & Q S^{-1} Q-A_{o}^{\top} Q \\
Q S^{-1} Q-Q A_{o} & 2 Q S^{-1} Q
\end{array}\right]} \\
& =\left[\begin{array}{cc}
X_{o} & \Gamma_{o}-A^{\top} Q \\
\Gamma_{o}-Q A & 2\left(\alpha Q+\Gamma_{o}\right)
\end{array}\right] \geq 0
\end{aligned}
$$

Furthermore, LMI (16) is equivalent through Schur complement to

$$
2 \alpha Q+2 \Gamma_{o}-\Theta_{o} \geq 0,
$$

with

$$
\Theta_{o}:=\left(\Gamma_{o}-Q A\right) X_{o}^{-1}\left(\Gamma_{o}-A^{\top} Q\right) .
$$

Note that there exists $\alpha$, large enough, such that (17) is satisfied. This completes the proof.

The results on generalized and extended observability Gramians have a controllability version as follows.

Theorem 2. (controllability Gramians)

The inequality (3) has a solution $\breve{P}>0$ if and only if the LMI (11) has a solution $(P, T, \beta)$ with $P>0$. Furthermore, if $X_{c}>0$, then there exists a $\beta>0$ large enough, and $T=T^{\top}>0$ such that the LMI (11) holds.

Proof. In order to establish the proof, note that (11) is equivalent to the following LMI

$$
\left[\begin{array}{cc}
X_{c} & Y_{c} \\
Y_{c}^{\top} & T+T^{\top}-T^{\top} B B^{\top} T
\end{array}\right] \geq 0 .
$$

Only if. Assume that (11) admits a solution $(P, T, \beta)$ with $P>0$, thus equivalently, (18) is satisfied. Multiplying the latter LMI by $\left[\begin{array}{lll}I_{n} & \mathbf{0}_{n \times n}\end{array}\right]$ from the left and by $\left[\begin{array}{ll}I_{n} & \mathbf{0}_{n \times n}\end{array}\right]^{\top}$ from the right, it follows that

$$
\begin{aligned}
& X_{c} \geq 0 \\
& \Longleftrightarrow-P A-A^{\top} P-P B B^{\top} P \geq 0, \\
& \Longleftrightarrow A \breve{P}+\breve{P} A^{\top}+B B^{\top} \leq 0
\end{aligned}
$$

where we used (9) to obtain the last inequality.

If. Assume there exists $\breve{P}>0$ solution to (3). Fix 1 ] $T=P\left(\beta I_{n}-A\right)^{-1}$, with $P$ defined in (9), then we get

$$
\begin{aligned}
Y_{c} & =-P+\left(A_{c}^{\top}+P B B^{\top}\right) P\left(\beta I_{n}-A\right)^{-1} \\
& =-P+\left(\beta P-P A-X_{c}\right)\left(\beta I_{n}-A\right)^{-1} \\
& =-X_{c}\left(\beta I_{n}-A\right)^{-1} \\
& =-X_{c} \breve{P} T
\end{aligned}
$$

and

\footnotetext{
${ }^{1}$ Since $\beta \geq 0$ and $\Re\{\lambda(A)\}<0, \beta$ is not an eigenvalue of $A$.
} 


$$
\begin{aligned}
T+T^{\top}-T^{\top} B B^{\top} T & =T^{\top}\left(T^{-1}+T^{-\top}-B B^{\top}\right) T \\
& =T^{\top} \breve{P}\left(2 \beta P+X_{c}\right) \breve{P} T .
\end{aligned}
$$

Hence, LMI (18) takes the form

$$
\left[\begin{array}{cc}
X_{c} & -X_{c} \breve{P} T \\
T^{\top} \breve{P} X_{c} & T^{\top} \breve{P}\left(2 \beta P+X_{c}\right) \breve{P} T
\end{array}\right] \geq 0 .
$$

Now, we multiply (22) by $\operatorname{diag}\left\{I_{n}, P T^{-\top}\right\}$ from the left, and by $\operatorname{diag}\left\{I_{n}, T^{-1} P\right\}$, yielding

$$
\left[\begin{array}{cc}
X_{c} & -X_{c} \\
-X_{c} & X_{c}
\end{array}\right]+\left[\begin{array}{cc}
\mathbf{0}_{n \times n} & \mathbf{0}_{n \times n} \\
\mathbf{0}_{n \times n} & 2 \beta P
\end{array}\right] \geq 0
$$

which holds for every $\beta \geq 0$.

Symmetric T. Assume that $P>0$ and $X_{c}>0$. Consider a symmetric matrix $\Gamma_{c} \in \mathbb{R}^{n \times n}$ verifying

$$
\beta \breve{P}+\Gamma_{c}>0 .
$$

Select

$$
T=\left(\beta \breve{P}+\Gamma_{c}\right)^{-1} .
$$

Hence, $T=T^{\top}>0$. Multiply (18) by $\operatorname{diag}\left\{I_{n}, T^{-\top}\right\}$ from the left and by $\operatorname{diag}\left\{I_{n}, T^{-1}\right\}$ from the right, and substitute (25) to obtain

$$
\left[\begin{array}{cc}
X_{c} & -P \Gamma_{c}+A^{\top}+P B B^{\top} \\
-\Gamma_{c} P+A+B B^{\top} P & 2\left(\beta \breve{P}+\Gamma_{c}\right)-B B^{\top}
\end{array}\right] \geq 0,
$$

which is equivalent to

$$
2 \beta \breve{P}+2 \Gamma_{c}-B B^{\top}-\Theta_{c} \geq 0
$$

where

$$
\Theta_{c}:=\left(-\Gamma_{c} P+A+B B^{\top} P\right) X_{c}^{-1}\left(-P \Gamma_{c}+A^{\top}+P B B^{\top}\right) .
$$

Since $\Theta_{c}$ does not depend on $\beta$, it follows that LMI (26), and in consequence LMI (11), holds for $\beta>0$ large enough. This completes the proof.

Remark 1. For clarity of presentation, we assume that $X_{o}>0, X_{c}>0$ to prove the existence of symmetric solutions to (10) and (11), respectively. While these conditions are not restrictive, they can be relaxed to $X_{o} \geq 0, X_{c} \geq 0$ by using generalized inverses. This however needs the introduction of the following conditions

$$
\begin{aligned}
\left(I_{n}-X_{o} X_{o}^{\dagger}\right)\left(\Gamma_{o}-A^{\top} Q\right) & =\mathbf{0}_{n \times n} \\
\left(I_{n}-X_{c} X_{c}^{\dagger}\right)\left(-P \Gamma_{c}+A^{\top}+P B B^{\top}\right) & =\mathbf{0}_{n \times n},
\end{aligned}
$$

where $X_{o}^{\dagger}, X_{c}^{\dagger}$ denote generalized inverses of $X_{o}$ and $X_{c}$, respectively. Note that both expressions in (28) are naturally satisfied if $X_{o}>0, X_{c}>0$. 
Remark 2. The symmetric matrices $\Gamma_{o}$ and $\Gamma_{c}$ provide degrees of freedom in the selection of the extended Gramians. These degrees of freedom can be used to improve the error bound in case the Gramians are used for model reduction, see Section 3.1, or to impose a desired structure to the reduced order model as is illustrated in Section 5 .

For the model reduction application, we assume that the matrices $S$ and $T$ are symmetric. From Theorems 1 and 2, it is clear that this assumption is not necessary to ensure the existence of solutions to (10) and (11), but we need it for obtaining an error bound in Section 3.1

In the extended balancing approach, a CTLTI system is said to be extended balanced if

$$
S=T^{-1}=\Lambda_{S T}
$$

where $\Lambda_{S T}$ is a diagonal matrix, see the Notation section. Therefore, we look for an invertible state transformation

$$
\bar{x}=W_{e}^{-1} x
$$

such that

$$
W_{e}^{-1} T^{-1} S W_{e}=\Lambda_{S T}^{2}
$$

Similar to Section 2.1] we assume that the elements of the diagonal matrix $\Lambda_{S T}$ are ordered from largest to smallest. Hence, the order of the CTLTI system is reduced by truncating the states that correspond to the smallest elements of the aforementioned matrix.

The discrete-time version of the LMIs (10) and (11) can be found in [4] and [5]. While, a thorough exposition of extended balanced truncation for discrete-time linear time-invariant (DTLTI) systems is given in [16] and 17].

\subsection{Computation of the error bound}

One of the appealing features of the balanced truncation approach is the possibility of establishing a clear error bound. For the generalized balanced truncation case, the inequality (8) establishes the error bound, which is customarily obtained through the analysis in the frequency domain of the original system and the reduced order one [10, 22. In this subsection we provide a procedure, different from the approach proposed in [19], to compute such error bound for the extended case. Towards this end, we assume that the linear transformation $W_{e}$, such that (30) holds, is known. Then, we introduce the following state-space systems

$$
\begin{gathered}
\bar{\Sigma}:\left\{\begin{array}{l}
\dot{\bar{x}}=\bar{A} \bar{x}+\bar{B} u \\
\bar{y}=\bar{C} \bar{x}
\end{array}\right. \\
\Sigma_{r}:\left\{\begin{array}{l}
\dot{x}_{r}=\bar{A} x_{r}+\bar{B} u+v(t) \\
y_{r}=\bar{C} x_{r},
\end{array}\right.
\end{gathered}
$$

where $\bar{x}$ is defined as in (29), $v(t) \in \mathbb{R}^{n}$ is an external signal, $x_{r} \in \mathbb{R}^{n}$ is an auxiliary state, and

$$
\bar{A}:=W_{e}^{-1} A W_{e}, \quad \bar{B}:=W_{e}^{-1} B, \quad \bar{C}:=C W_{e} .
$$


Now, we split $\bar{x}$ system into two parts, namely,

$$
\bar{x}=\left[\begin{array}{l}
\bar{x}_{1} \\
\bar{x}_{2}
\end{array}\right],
$$

where $\bar{x}_{1} \in \mathbb{R}^{k}$ is the part of the state to be preserved after the reduction of the model and $\bar{x}_{2} \in \mathbb{R}^{\ell}$, with $\ell:=n-k$, is the part to be truncated. Accordingly, the matrices given in (33) can be expressed as follows

$$
\bar{A}=\left[\begin{array}{ll}
\bar{A}_{11} & \bar{A}_{12} \\
\bar{A}_{21} & \bar{A}_{22}
\end{array}\right], \quad \bar{B}=\left[\begin{array}{l}
\bar{B}_{1} \\
\bar{B}_{1}
\end{array}\right], \quad \bar{C}=\left[\begin{array}{l}
\bar{C}_{1} \\
\bar{C}_{2}
\end{array}\right],
$$

with

$$
\begin{array}{llll}
\bar{A}_{11} \in \mathbb{R}^{k \times k}, & \bar{A}_{12} \in \mathbb{R}^{k \times \ell}, & \bar{A}_{21} \in \mathbb{R}^{\ell \times k}, & \bar{A}_{22} \in \mathbb{R}^{\ell \times \ell}, \\
\bar{B}_{1} \in \mathbb{R}^{k \times m}, & \bar{B}_{2} \in \mathbb{R}^{\ell \times m}, & \bar{C}_{1} \in \mathbb{R}^{q \times k}, & \bar{C}_{2} \in \mathbb{R}^{q \times \ell} .
\end{array}
$$

Thus, the truncation of the state $\bar{x}_{2}$ leads to the following reduced order model

$$
\widehat{\Sigma}:\left\{\begin{aligned}
\dot{\hat{x}} & =\widehat{A} \hat{x}+\widehat{B} u \\
\hat{y} & =\widehat{C} \hat{x}
\end{aligned}\right.
$$

where

$$
\hat{x}=\bar{x}_{1}, \quad \widehat{A}:=\bar{A}_{11}, \quad \widehat{B}:=\bar{B}_{1}, \quad \widehat{C}:=\bar{C}_{1} .
$$

Now, inspired by the ideas presented in [21], and by the approach adopted in [16], 17] for discrete-time, and in [19] for continuous-time, we propose a storage function that is instrumental to establish the error bound. Towards this end, we first introduce the following definitions to simplify the notation of this section:

$$
\begin{array}{ll}
\bar{Q}:=W_{e}^{\top} Q W_{e}, & \bar{P}:=W_{e}^{\top} P W_{e}, \\
z_{o}:=\bar{x}-x_{r}, & z_{c}:=\bar{x}+x_{r} .
\end{array}
$$

where $P$ is defined as in (9). The proposition below introduces a storage function which is used to establish the error bound in this section.

Proposition 1. Consider the systems $\Sigma, \bar{\Sigma}, \Sigma_{r}$ given in (11), (31), and (32), respectively. Assume that the triplet $(Q, S, \alpha)$ solves $L M I$ (10) and the triplet $(P, T, \beta)$ solves LMI (11). Consider the storage function

$$
\mathcal{S}\left(z_{o}, z_{c}\right)=z_{o}^{\top} \bar{Q} z_{o}+\sigma_{n}^{2} z_{c}^{\top} \bar{P} z_{c}
$$

where $\sigma_{n}$ is the $n-t h$ entry of $\Lambda_{S T}$, and $z_{o}, z_{c}$ are defined in (36). Then,

$$
\begin{aligned}
\dot{\mathcal{S}} \leq & 4 \sigma_{n}^{2}|u|^{2}-\left|y-y_{r}\right|^{2} \\
& +2\left[\sigma_{n}^{2}\left(\beta z_{c}+\dot{z}_{c}\right)^{\top} \Lambda_{S T}^{-1}-\left(\alpha z_{o}+\dot{z}_{o}\right)^{\top} \Lambda_{S T}\right] v
\end{aligned}
$$

Proof. Note that

$$
\dot{\mathcal{S}}=2 z_{o}^{\top} \bar{Q} \dot{z}_{o}+2 \sigma_{n}^{2} z_{c}^{\top} \bar{P} \dot{z}_{c}
$$

Define the vectors

$$
\xi_{o}:=\left[\begin{array}{c}
W_{e} z_{o} \\
W_{e} v
\end{array}\right], \quad \xi_{c}:=\left[\begin{array}{c}
W_{e} z_{c} \\
W_{e} v \\
u
\end{array}\right] .
$$


Multiply LMI (10) by $\xi_{o}^{\top}$ from the left and by $\xi_{o}$ from the right, yielding

$$
\begin{aligned}
& 2\left[v^{\top}-z_{o}^{\top}\left(\alpha I_{n}+\bar{A}^{\top}\right)\right] \Lambda_{S T} v \\
& +z_{o}^{\top}\left[W_{e}^{\top} X_{o} W_{e} z_{o}+2 \bar{Q} v\right] \geq 0 \\
& \Longleftrightarrow-2\left(\dot{z}_{o}+\alpha z_{o}\right)^{\top} \Lambda_{S T} v \\
& +z_{o}^{\top}\left[W_{e}^{\top} X_{o} W_{e} z_{o}+2 \bar{Q} v\right] \geq 0 \\
& \Longleftrightarrow-2\left(\dot{z}_{o}+\alpha z_{o}\right)^{\top} \Lambda_{S T} v-z_{o}^{\top} \bar{C}^{\top} \bar{C} z_{o} \\
& +2 z_{o}^{\top} \bar{Q}\left[v-\bar{A} z_{o}\right] \geq 0 \\
& \Longleftrightarrow-2\left(\dot{z}_{o}+\alpha z_{o}\right)^{\top} \Lambda_{S T} v-\left|y-y_{r}\right|^{2}-2 z_{o}^{\top} \bar{Q} \dot{z}_{o} \geq 0,
\end{aligned}
$$

where we used the facts

$$
\begin{aligned}
\dot{z}_{o} & =\bar{A} z_{o}-v \\
\bar{C} z_{o} & =y-y_{r} .
\end{aligned}
$$

Note that (41) implies that

$$
2 z_{o}^{\top} \bar{Q} \dot{z}_{o} \leq-2\left(\dot{z}_{o}+\alpha z_{o}\right)^{\top} \Lambda_{S T} v-\left|y-y_{r}\right|^{2} .
$$

Now, multiply LMI (11) by $\xi_{c}^{\top}$ from the left and by $\xi_{c}$ from the right to obtain

$$
\begin{aligned}
& -2 z_{c}^{\top} \bar{P}\left[\bar{A} z_{c}+2 \bar{B} u+v\right]+4|u|^{2} \\
& +2\left[z_{c}^{\top}\left(\beta I_{n}+\bar{A}^{\top}\right)+v^{\top}+2 u^{\top} \bar{B}^{\top}\right] \Lambda_{S T}^{-1} v \geq 0 \\
& \Longleftrightarrow-2 z_{c}^{\top}\left(\bar{A} z_{c}+2 \bar{B} u+v\right)+4|u|^{2} \\
& \quad+2\left(\dot{z}_{c}+\beta z_{c}\right)^{\top} \Lambda_{S T}^{-1} v \geq 0 \\
& \quad \Longleftrightarrow 4|u|^{2}+2\left(\dot{z}_{c}+\beta z_{c}\right)^{\top} \Lambda_{S T}^{-1} v \geq 2 z_{c}^{\top} \bar{P} \dot{z}_{c},
\end{aligned}
$$

where we used that

$$
\dot{z}_{c}=\bar{A} z_{c}+2 \bar{B} u+v .
$$

The proof is completed by substituting (43) and (44) in (39) to obtain (38).

In order to establish the error bound, we propose a particular selection of the signal $v(t)$ that allow us to compare the behavior of systems (31) and (32).

Lemma 1. Consider $\ell=1$. Assume that systems (32) and (35) are initially at rest. Consider the partition $x_{r}=\left[\begin{array}{ll}x_{r_{1}}^{\top} & x_{r_{2}}^{\top}\end{array}\right]^{\top}$, with $x_{r_{1}} \in \mathbb{R}^{n-1}$ and $x_{r_{2}} \in \mathbb{R}$. Choose

$$
v(t)=-\left[\begin{array}{c}
\mathbf{0}_{n-1} \\
\bar{A}_{21} x_{r_{1}}(t)+\bar{B}_{2} u(t)
\end{array}\right] .
$$

Then, $\hat{y}(t)=y_{r}(t)$, and $x_{r_{2}}(t)=0$ for every $t \geq 0$.

Proof. To establish the proof replace (46) in (32) to obtain

$$
\begin{aligned}
& \dot{x}_{r_{1}}=\bar{A}_{11} x_{r_{1}}+\bar{A}_{12} x_{r_{2}}+\bar{B}_{1} u \\
& \dot{x}_{r_{2}}=\bar{A}_{22} x_{r_{2}}
\end{aligned}
$$


Since $x_{r}(0)=\mathbf{0}_{n}$, from (47) we have the following chain of implications

$$
\begin{aligned}
\dot{x}_{r_{2}}=0 \forall t \geq 0 & \Longrightarrow x_{r_{2}}(t)=0 \forall t \geq 0 \\
& \Longrightarrow \dot{x}_{r_{1}}=\bar{A}_{11} x_{r_{1}}+\bar{B}_{1} u .
\end{aligned}
$$

Since $\hat{x}(0)=\mathbf{0}_{n-1}$, the last expression of (48) implies that $\hat{x}(t)=x_{r_{1}}(t)$ for all $t \geq 0$. Hence,

$$
y_{r}=\bar{C}_{1} x_{r_{1}}=\widehat{C} \hat{x}=\hat{y} .
$$

Using the results of Proposition 1 and Lemma 1 the following Lemma establishes an error bound for the case $\ell=1$, that is, when only one state is truncated.

Lemma 2. Consider the balanced system (31) with extended observability Gramian $\left(\bar{Q}, \Lambda_{S T}, \alpha\right)$, and inverse extended controllability Gramian $\left(\bar{P}, \Lambda_{S T}^{-1}, \beta\right)$, where $\alpha=\beta$ and $\ell=1$. Assume that systems (60), (35) and (32) are initially at rest and select $v$ as in (46). Then,

$$
\|\Sigma-\widehat{\Sigma}\|_{\infty} \leq 2 \sigma_{n} .
$$

Proof. Define

$$
v_{2}:=\bar{A}_{21} x_{r_{1}}(t)+\bar{B}_{2} u(t) .
$$

Hence, we can rewrite (46) as follows

$$
v=\left[\begin{array}{c}
\mathbf{0}_{n-1} \\
v_{2}
\end{array}\right] .
$$

On the other hand, from Lemma 1 we have that

$$
x_{r}=\left[\begin{array}{c}
\hat{x} \\
0
\end{array}\right], \quad y_{r}=\hat{y} .
$$

Therefore, since $\alpha=\beta$, we get

$$
\begin{aligned}
\left(\alpha z_{o}+\dot{z}_{o}\right)^{\top} \Lambda_{S T} v & =\sigma_{n}\left(\alpha \bar{x}_{2}+\dot{\bar{x}}_{2}\right) v_{2} \\
& =\sigma_{n}^{2}\left(\beta z_{c}+\dot{z}_{c}\right)^{\top} \Lambda_{S T}^{-1} v .
\end{aligned}
$$

Now, consider the storage function $\mathcal{S}\left(z_{o}, z_{c}\right)$, given in (37). Then, substituting (54) in (38), its derivative along the trajectories reduces to

$$
\dot{\mathcal{S}} \leq 4 \sigma_{n}^{2}|u|^{2}-|y-\hat{y}|^{2},
$$

where we used (54). Moreover, integrating (55) from 0 to $\infty$, yields

$$
0 \leq 4 \sigma_{n}^{2}\|u\|_{2}^{2}-\|y-\hat{y}\|_{2}^{2}
$$

which implies

$$
\|y-\hat{y}\|_{2} \leq 2 \sigma_{n}\|u\|_{2} \text {. }
$$

The proof is completed by using the induced $\mathcal{L}_{2}$ norm, see Proposition 5.13 and the table on page 150 of [1]. 
Now we are in position to present the main result of this paper in terms of the error bound for model reduction for CTLTI systems based on extended balanced truncation.

Theorem 3. Consider the balanced system (31) with extended observability Gramian $\left(\bar{Q}, \Lambda_{S T}, \alpha\right)$, and inverse extended controllability $\operatorname{Gramian}\left(\bar{P}, \Lambda_{S T}^{-1}, \beta\right)$, where $\alpha=\beta$ and

$$
\Lambda_{S T}=\operatorname{diag}\left\{\sigma_{1}, \cdots, \sigma_{n}\right\} .
$$

Consider the truncated $k^{\text {th }}$ order system (35). Then, the error bound is given by the following inequality

$$
\|\Sigma-\widehat{\Sigma}\|_{\infty} \leq 2 \sum_{j=k+1}^{n} \sigma_{j} .
$$

Proof. To establish the proof apply iteratively Lemma 2

Remark 3. If the matrices $\Gamma_{o}$ and $\Gamma_{c}$ are chosen as zero and $\alpha=\beta$, then $S=\frac{1}{\alpha} Q$ and $T=\frac{1}{\alpha} P$. Hence, $Q \breve{P}=S T^{-1}$, and $\Lambda_{Q P}=\Lambda_{S T}$. Accordingly, the error bound obtained via extended balancing coincides with the error bound obtained from the generalized balancing approach. Moreover, the reduced-order model obtained from both methods is the same.

Similar to the discrete-time results reported in [16] and [17, the error bound (58) is obtained by proposing a storage function and using dissipativity arguments, as in [21]. This procedure contrasts to the traditional analysis using transfer functions.

\section{Balancing of CTLTI PH systems}

From now onwards, we focus on the study of $\mathrm{PH}$ systems. These systems have been proved to be suitable to capture physical phenomena in different domains while preserving conservation laws [6], [20]. In this framework, it is possible to represent large scale networks of complex physical systems and, at the same time, underscore the roles of the energy, the interconnection pattern, and the dissipation in the behavior of such systems. Moreover, the passivity property of these systems can be straightforwardly proved by selecting the Hamiltonian function as a storage function. Thus, given the possible physical interpretation of the PH models and their geometrical properties, this framework is appealing from both points of view: the theoretical and the practical one. Therefore, preserving the $\mathrm{PH}$ structure for the reduced order model is interesting for analysis purposes and might be useful to give an interpretation of the behavior of the reduced order system. In this section, we aim to solve the model reduction problem of CTLTI PH systems while preserving the $\mathrm{PH}$ structure for the reduced order system. Furthermore, in some cases, not only the PH structure is preserved, but more particular structures which permit to provide a physical interpretation of the reduced order model. 


\subsection{CTLTI PH systems}

The representation of a CTLTI PH system is given by

$$
\Sigma_{H}:\left\{\begin{aligned}
\dot{x} & =(J-R) H x+B u \\
y & =B^{\top} H x \\
\mathcal{H}(x) & =\frac{1}{2} x^{\top} H x
\end{aligned}\right.
$$

where $x \in \mathbb{R}^{n}$ is the state vector, $u, y \in \mathbb{R}^{m}$ are the input and output vectors, respectively, $\mathcal{H}(x)$ represents the Hamiltonian of the system, with $H=H^{\top}>0$; and $R=R^{\top} \geq 0, J=-J^{\top}$ represent the dissipation and the interconnection matrix, respectively. In order to simplify notation, we define $F:=J-R$.

The objective of this work is twofold: on the one hand, we aim to balance system (59) and obtain a lower order model. On the other hand, we want the reduced model to have a $\mathrm{PH}$ structure because of the interpretation and the interconnection properties of this kind of systems. Towards this end, we assume that system (59) is asymptotically stable and we look for an invertible linear transformation $W$ that balances the system. Such transformation is given by $W=W_{g}$ in the generalized case, while in the extended case we have $W=W_{e}$. Then, we write the dynamics of the balanced system as follows

$$
\bar{\Lambda}_{H}:\left\{\begin{aligned}
\dot{\bar{x}} & =\bar{F} \bar{H} \bar{x}+\bar{B} u \\
\bar{y} & =\bar{B}^{\top} \bar{H} \bar{x}
\end{aligned}\right.
$$

where

$$
\bar{F}:=W^{-1} F W^{-\top}, \quad \bar{H}:=W^{\top} H W, \quad \bar{B}:=W^{-1} B .
$$

Hence, if we split $\bar{x}$ as in (34), the balanced system can be expressed as

$$
\bar{\Sigma}_{H}:\left\{\begin{aligned}
{\left[\begin{array}{c}
\dot{\bar{x}}_{1} \\
\overline{\bar{x}}_{2}
\end{array}\right] } & =\left[\begin{array}{ll}
\bar{F}_{11} & \bar{F}_{12} \\
\bar{F}_{21} & \bar{F}_{22}
\end{array}\right]\left[\begin{array}{ll}
\bar{H}_{11} & \bar{H}_{12} \\
\bar{H}_{12}^{\top} & \bar{H}_{22}
\end{array}\right]\left[\begin{array}{l}
\bar{x}_{1} \\
\bar{x}_{2}
\end{array}\right]+\left[\begin{array}{l}
\bar{B}_{1} \\
\bar{B}_{2}
\end{array}\right] u \\
\bar{y} & =\left[\begin{array}{ll}
\bar{B}_{1}^{\top} & \bar{B}_{2}^{\top}
\end{array}\right]\left[\begin{array}{ll}
\bar{H}_{11} & \bar{H}_{12} \\
\bar{H}_{12}^{\top} & \bar{H}_{22}
\end{array}\right]\left[\begin{array}{l}
\bar{x}_{1} \\
\bar{x}_{2}
\end{array}\right],
\end{aligned}\right.
$$

with

$$
\begin{array}{lll}
\bar{F}_{11}, \bar{H}_{11} \in \mathbb{R}^{k \times k}, & \bar{F}_{22}, \bar{H}_{22} \in \mathbb{R}^{\ell \times \ell}, & \bar{F}_{12}, \bar{H}_{12} \in \mathbb{R}^{k \times \ell}, \\
\bar{B}_{1} \in \mathbb{R}^{k \times m}, & \bar{F}_{21} \in \mathbb{R}^{\ell \times k}, & \bar{B}_{2} \in \mathbb{R}^{\ell \times m} .
\end{array}
$$

Problem formulation for PH systems. Given the system (59), find an invertible linear transformation $W$, that performs the balancing of the system and at the same time satisfies

$$
\bar{H}_{12}=\mathbf{0}_{k \times n-k} \text {. }
$$

Note that, if (61) holds, the truncation leads to the following reduced order system

$$
\widehat{\Sigma}_{H}:\left\{\begin{aligned}
\dot{\hat{x}} & =\bar{F}_{11} \bar{H}_{11} \hat{x}+\bar{B}_{1} u \\
\hat{y} & =\bar{B}_{1}^{\top} \bar{H}_{11} \hat{x} \\
\hat{\mathcal{H}}(\hat{x}) & =\frac{1}{2} \hat{x}^{\top} \bar{H}_{11} \hat{x}
\end{aligned}\right.
$$


which is another CTLTI PH system, with $\hat{x}=\bar{x}_{1}$. Therefore, it follows that one solution to the problem of model reduction with $\mathrm{PH}$ structure preservation takes place when the Hamiltonian matrix of the balanced system, $\bar{H}$, is diagonal. In such case, our problem is reduced to the simultaneous diagonalization of three matrices, namely, $(Q, P, H)$ or $(S, T, H)$.

Remark 4. The complete diagonalization of $H$ is not necessary. In fact, a block diagonalization that ensures (61) is enough to preserve the PH structure. Nevertheless, if $H$ is not a diagonal matrix, then it is necessary to know the dimension of the part of the state to be truncated.

The subsequent sections of this paper are devoted to the identification of a transformation $W$ that balances the system and ensures that (61) is satisfied.

\subsection{Generalized balancing of CTLTI PH systems}

In this subsection, we study the generalized balancing method for CTLTI PH systems which is the starting point of extended balancing of CTLTI PH studied in Section 4.3. Below, we provide sufficient conditions to ensure the existence of a transformation $W_{g}$ that complies with the requirements established in Section 4.1. To this end, we revisit the following theorem which establishes necessary and sufficient conditions for the existence of a transformation that diagonalizes simultaneously three matrices when at least one of them has definite sign.

Theorem 4 (15]). Let $L, M, N$ be symmetric matrices. In the case of at least one fixed-sign quadratic form (e.g., $M$ positive definite), the condition

$$
L M^{-1} N=N M^{-1} L
$$

is necessary and sufficient for the existence of a linear invertible congruent transformation $W$ that diagonalizes simultaneously $L, M$ and $N$.

For the proof and further details about Theorem 4, we refer the reader to [15] and [2]. For a thorough exposition on simultaneously diagonalizable matrices, we refer the reader to [12, Chapter 4.

In generalized balancing of CTLTI PH systems, the condition (63) takes the form

$$
H \breve{P}^{-1} Q=Q \breve{P}^{-1} H .
$$

Accordingly, we look for $Q$ and $\breve{P}$ verifying (2) and (3), respectively, such that (64) holds. A trivial solution to this problem takes place when $Q$ or $\breve{P}$ coincides with the (scaled) Hamiltonian matrix $H$ or its inverse. This idea has been studied in 8 8 and [13, among other works; and for the sake of completeness, the proposition below identifies a class of CTLTI PH systems for which the (scaled) Hamiltonian matrix, or its inverse, solves the inequalities (21) and (3).

Proposition 2. Consider $\delta \in \mathbb{R}_{>0}$. Assume that the system (59) is asymptotically stable. If the following condition holds

$$
2 \delta R-B B^{\top} \geq 0 .
$$

Then $Q=\delta H$ solves (21) and $\breve{P}=\delta H^{-1}$ is a solution to (3). 
Proof. To establish the proof note that for CTLTI PH systems (2) and (3) take the form

$$
\begin{aligned}
Q F H+H F^{\top} Q+H B B^{\top} H & \leq 0 \\
F H \breve{P}+\breve{P} H F^{\top}+B B^{\top} & \leq 0,
\end{aligned}
$$

respectively. Hence, substituting $Q=\delta H$ in (66), we obtain

$$
\begin{aligned}
0 & \geq \delta H F H+\delta H F^{\top} H+H B B^{\top} H \\
& =H\left(B B^{\top}-2 \delta R\right) H \\
\Longleftrightarrow 0 & \leq 2 \delta R-B B^{\top} .
\end{aligned}
$$

On the other hand, replacing $\breve{P}=\delta H^{-1}$ in (67), we have

$$
\begin{aligned}
0 & \geq \delta F+\delta F^{\top}+B B^{\top} \\
& =-2 \delta R+B B^{\top} \\
\Longleftrightarrow 0 & \leq 2 \delta R-B B^{\top} .
\end{aligned}
$$

Condition (65) is satisfied by systems that have dissipation in all the input channels, e.g., fully damped mechanical systems. Nonetheless, $R$ and $B$ are system parameters, thus, it might happen that condition (65) is not satisfied by the system (59). In order to overcome this issue, below we state two propositions to identify generalized Gramians such that the triplet $(Q, \breve{P}, H)$ verifies (64) and solves the Lyapunov inequalities (66) and (67). These propositions represent the main result of this paper in terms of generalized balancing with $\mathrm{PH}$ structure preservation

Proposition 3. Let $\breve{P}$ be a solution to (67). Consider a full rank matrix $\phi_{P} \in$ $\mathbb{R}^{n \times n}$ verifying the following

$$
\begin{aligned}
\breve{P} & =\phi_{P}^{\top} \phi_{P} \\
\phi_{P} H \phi_{P}^{\top} & =U_{H P} \Lambda_{H P} U_{H P}^{\top},
\end{aligned}
$$

where $U_{H P}$ is an orthogonal matrix, and $\Lambda_{H P}$ is a diagonal matrix whose entries are the singular values of $\phi_{P} H \phi_{P}^{\top}$, see the notation at the end of Section 1 . Define the matrices

$$
\begin{aligned}
\mathcal{F}_{c} & :=U_{H P}^{\top} \phi_{P}^{-\top} F \phi_{P}^{-1} U_{H P} \\
\mathcal{B}_{c} & :=U_{H P}^{\top} \phi_{P}^{-\top} B .
\end{aligned}
$$

Assume that

$$
-\Lambda_{Q P}^{2} \Lambda_{H P}^{-1} \mathcal{F}_{c}-\mathcal{F}_{c}^{\top} \Lambda_{H P}^{-1} \Lambda_{Q P}^{2}-\mathcal{B}_{c} \mathcal{B}_{c}^{\top} \geq 0
$$

holds for a diagonal matrix $\Lambda_{Q P}$. Hence, (66) is solved by

$$
Q=\phi_{P}^{-1} U_{H P} \Lambda_{Q P}^{2} U_{H P}^{\top} \phi_{P}^{-\top} .
$$

Moreover, the transformation

$$
W_{g c}=\phi_{P}^{\top} U_{H P} \Lambda_{Q P}^{-\frac{1}{2}}
$$

balances the system and diagonalizes $H$. 
Proof. To establish the proof we define

$$
\mathcal{X}_{o}:=-\Lambda_{Q P}^{2} \Lambda_{H P}^{-1} \mathcal{F}_{c}-\mathcal{F}_{c}^{\top} \Lambda_{H P}^{-1} \Lambda_{Q P}^{2}-\mathcal{B}_{c} \mathcal{B}_{c}^{\top} .
$$

Note that, if (69) holds, we have the following chain of implications

$$
\begin{aligned}
\mathcal{X}_{o} & \geq 0 \\
\Longleftrightarrow \phi_{P}^{-1} U_{H P} \Lambda_{H P} \mathcal{X}_{o} \Lambda_{H P} U_{H P}^{\top} \phi_{P}^{-\top} & \geq 0 \\
\Longleftrightarrow-Q F H-H F^{\top} Q-H B B^{\top} H & \geq 0 \\
\Longleftrightarrow Q F H+H F^{\top} Q+H B B^{\top} H & \leq 0
\end{aligned}
$$

where we used (68) and (70). Moreover,

$$
\begin{aligned}
W_{g c}^{\top} Q W_{g c} & =\Lambda_{Q P} \\
W_{g c}^{-1} \breve{P} W_{g c}^{-\top} & =\Lambda_{Q P} \\
W_{g c}^{\top} H W_{g c} & =\Lambda_{Q P}^{-1} \Lambda_{H P} .
\end{aligned}
$$

This completes the proof.

The following proposition is the dual version of Proposition 3 and relaxes condition (65), in this case, for a given generalized observability Gramian $Q$.

Proposition 4. Let $Q$ be a solution to (66). Consider a full rank matrix $\phi_{Q} \in$ $\mathbb{R}^{n \times n}$ verifying the following

$$
\begin{aligned}
Q & =\phi_{Q}^{\top} \phi_{Q} \\
\phi_{Q}^{-\top} H \phi_{Q}^{-1} & =U_{H Q} \Lambda_{H Q} U_{H Q}^{\top} .
\end{aligned}
$$

Define the matrices

$$
\begin{aligned}
\mathcal{F}_{o} & :=U_{H Q}^{\top} \phi_{Q} F \phi_{Q}^{\top} U_{H Q} \\
\mathcal{B}_{o} & :=U_{H Q}^{\top} \phi_{Q} B .
\end{aligned}
$$

Assume that

$$
-\mathcal{F}_{o} \Lambda_{H Q} \Lambda_{Q P}^{2}-\Lambda_{Q P}^{2} \Lambda_{H Q} \mathcal{F}_{o}^{\top}-\mathcal{B}_{o} \mathcal{B}_{o}^{\top} \geq 0
$$

holds for a diagonal matrix $\Lambda_{Q P}$. Hence, (67) is solved by

$$
\breve{P}=\phi_{Q}^{-1} U_{H Q} \Lambda_{Q P}^{2} U_{H Q}^{\top} \phi_{Q}^{-\top} .
$$

Moreover, the transformation

$$
W_{g o}=\phi_{Q}^{-1} U_{H Q} \Lambda_{Q P}^{\frac{1}{2}}
$$

balances the system and diagonalizes $H$.

Proof. Define

$$
\mathcal{X}_{c}:=-\mathcal{F}_{o} \Lambda_{H Q} \Lambda_{Q P}^{2}-\Lambda_{Q P}^{2} \Lambda_{H Q} \mathcal{F}_{o}^{\top}-\mathcal{B}_{o} \mathcal{B}_{o}^{\top} .
$$


Therefore, if (73) is satisfied, we have

$$
\begin{aligned}
\mathcal{X}_{c} & \geq 0 \\
\Longleftrightarrow \phi_{Q}^{-1} U_{H Q} \mathcal{X}_{c} U_{H Q}^{\top} \phi_{Q}^{-\top} & \geq 0 \\
\Longleftrightarrow-F H \breve{P}-\breve{P} H F^{\top}-B B^{\top} & \geq 0 \\
\Longleftrightarrow F H \breve{P}+\breve{P} H F^{\top}+B B^{\top} & \leq 0,
\end{aligned}
$$

where we used (72) and (74). To complete the proof, note that

$$
\begin{aligned}
W_{g o}^{\top} Q W_{g o} & =\Lambda_{Q P} \\
W_{g o}^{-1} \breve{P} W_{g o}^{-\top} & =\Lambda_{Q P} \\
W_{g o}^{\top} H W_{g o} & =\Lambda_{H Q} \Lambda_{Q P} .
\end{aligned}
$$

In Propositions 3 and 4 the condition (65) is relaxed by imposing a particular structure to the generalized observability and controllability Gramians, respectively. Such structure depends on the Hamiltonian matrix, however, it is less restrictive than (65). Indeed, if this latter condition is satisfied, then (69) and (73) hold.

Using the results presented in this section, below we study extended balancing of CTLTI PH systems. As was mentioned in Section 3, the use of extended Gramians can be advantageous for different purposes, for instance, to obtain a lower error bound or to impose a more particular structure to the reduced order model.

\subsection{Extended balancing of CTLTI PH systems}

Similar to the generalized balancing case, in this section we provide sufficient conditions for the existence of a linear transformation $W_{e}$ that balances the system and diagonalizes the Hamiltonian matrix. Towards this end, below we introduce two propositions that provide a suitable transformation $W_{e}$. Such propositions constitute the main result of this work regarding extended balancing with $\mathrm{PH}$ structure preservation.

Proposition 5. Let $\breve{P}$ be a solution to (67) such that $X_{c}>0$. Select $\beta$ and $\Gamma_{c}$ such that (24) holds and T, defined in (25), solves LMI (11). Consider a full rank matrix $\phi_{T} \in \mathbb{R}^{n \times n}$ verifying the following

$$
\begin{aligned}
T^{-1} & =\phi_{T}^{\top} \phi_{T} \\
\phi_{T} H \phi_{T}^{\top} & =U_{H T} \Lambda_{H T} U_{H T}^{\top} .
\end{aligned}
$$

Define the matrices

$$
\begin{aligned}
\mathcal{F}_{e c} & :=U_{H T}^{\top} \phi_{T}^{-\top} F \phi_{T}^{-1} U_{H T} \\
\mathcal{B}_{e c} & :=U_{H T}^{\top} \phi_{T}^{-\top} B .
\end{aligned}
$$

Assume that

$$
-\Lambda_{Q T}^{2} \Lambda_{H T}^{-1} \mathcal{F}_{e c}-\mathcal{F}_{e c}^{\top} \Lambda_{H T}^{-1} \Lambda_{Q T}^{2}-\mathcal{B}_{e c} \mathcal{B}_{e c}^{\top}>0
$$


holds for a diagonal matrix $\Lambda_{Q T}$. Then, (66) is solved by

$$
Q=\phi_{T}^{-1} U_{H T} \Lambda_{Q T}^{2} U_{H T}^{\top} \phi_{T}^{-\top} .
$$

Select $\alpha$ such that the matrix

$$
S=\frac{1}{\alpha} Q
$$

solves LMI (10). Then, the invertible transformation

$$
W_{e c}=\sqrt[4]{\alpha} \phi_{T}^{\top} U_{H T} \Lambda_{Q T}^{-\frac{1}{2}}
$$

balances the system and diagonalizes $H$.

Proof. Define

$$
\mathcal{X}_{e o}:=-\Lambda_{Q T}^{2} \Lambda_{H T}^{-1} \mathcal{F}_{e c}-\mathcal{F}_{e c}^{\top} \Lambda_{H T}^{-1} \Lambda_{Q T}^{2}-\mathcal{B}_{e c} \mathcal{B}_{e c}^{\top} .
$$

Then, the inequality (78) is satisfied if and only if

$$
\begin{aligned}
\mathcal{X}_{e o} & >0 \\
\phi_{T}^{-1} U_{H T} \Lambda_{H T} \mathcal{X}_{e o} \Lambda_{H T} U_{H T}^{\top} \phi_{T}^{-\top} & >0 \\
\Longleftrightarrow X_{o} & >0 \\
\Longleftrightarrow Q F H+H F^{\top} Q+H B B^{\top} H & <0 \\
\Longleftrightarrow Q F H+H F^{\top} Q+H B B^{\top} H & \leq 0,
\end{aligned}
$$

where we used

$$
A=F H \text {. }
$$

Fix $\Gamma_{o}=\mathbf{0}_{n \times n}$ in (15). Hence, for $\alpha$ large enough, the selection of $S$ given in (80) ) solves the LMI (10).

To establish the last part of the proof define

$$
\Lambda_{S T}:=\frac{1}{\sqrt{\alpha}} \Lambda_{Q T}
$$

note that

$$
\begin{aligned}
W_{e c}^{-1} T^{-1} S W_{e c} & =\Lambda_{S T}^{2} \\
W_{e c}^{\top} H W_{e c} & =\Lambda_{H T} \Lambda_{S T}^{-1} .
\end{aligned}
$$

The following proposition is the dual version of Proposition 5

Proposition 6. Let $Q$ be a solution to (66) such that $X_{o}>0$. Select $\alpha$ and $\Gamma_{o}$ such that (14) holds and $S$, defined in (15), solves LMI (10). Consider a full rank matrix $\phi_{S} \in \mathbb{R}^{n \times n}$ verifying the following

$$
\begin{aligned}
S & =\phi_{S}^{\top} \phi_{S} \\
\phi_{S}^{-\top} H \phi_{S}^{-1} & =U_{H S} \Lambda_{H S} U_{H S}^{\top}
\end{aligned}
$$

Define the matrices

$$
\begin{aligned}
\mathcal{F}_{e o} & :=U_{H S}^{\top} \phi_{S} F \phi_{S}^{\top} U_{H S} \\
\mathcal{B}_{e o} & :=U_{H S}^{\top} \phi_{S} B .
\end{aligned}
$$


Assume

$$
-\mathcal{F}_{\text {eo }} \Lambda_{H S} \Lambda_{S P}^{2}-\Lambda_{S P}^{2} \Lambda_{H S} \mathcal{F}_{\text {eo }}^{\top}-\mathcal{B}_{\text {eo }} \mathcal{B}_{\text {eo }}^{\top}>0
$$

holds for a diagonal matrix $\Lambda_{S P}$. Thus, (67) is solved by

$$
\breve{P}=\phi_{S}^{-1} U_{H S} \Lambda_{S P}^{2} U_{H S}^{\top} \phi_{S}^{-\top}
$$

Select $\beta$ such that the matrix

$$
T^{-1}=\beta \breve{P}
$$

solves LMI (11). Then,

$$
W_{e o}=\sqrt[4]{\beta} \phi_{S}^{-1} U_{H S} \Lambda_{S P}^{\frac{1}{2}}
$$

balances the system and diagonalizes $H$.

Proof. Define

$$
\mathcal{X}_{e o}:=-\mathcal{F}_{e o} \Lambda_{H S} \Lambda_{S P}^{2}-\Lambda_{S P}^{2} \Lambda_{H S} \mathcal{F}_{e o}^{\top}-\mathcal{B}_{e o} \mathcal{B}_{e o}^{\top} .
$$

Hence, if (87) holds, we have the following chain of implications

$$
\begin{aligned}
\mathcal{X}_{e o} & >0 \\
\Longleftrightarrow \phi_{S}^{-1} U_{H S} \mathcal{X}_{c o} U_{H S}^{\top} \phi_{S}^{\top} & >0 \\
\Longleftrightarrow-F H \breve{P}-\breve{P} H F^{\top}-B B^{\top} & >0 .
\end{aligned}
$$

Moreover,

$$
\begin{aligned}
-F H \breve{P}-\breve{P} H F^{\top}-B B^{\top}>0 \\
\Longrightarrow\left\{\begin{array}{r}
F H \breve{P}+\breve{P} H F^{\top}+B B^{\top} \leq 0 \\
X_{c}>0
\end{array}\right.
\end{aligned}
$$

where we used (83). Fix $\Gamma_{c}=\mathbf{0}_{n \times n}$ in (25). Accordingly, for $\beta$ large enough, the selection of $T$ given in (89) solves the LMI (11).

To establish the last part of the proof define

$$
\Lambda_{S T}:=\sqrt{\beta} \Lambda_{S P}
$$

Note that

$$
\begin{aligned}
W_{e o}^{-1} T^{-1} S W_{e o} & =\Lambda_{S T}^{2} \\
W_{e o}^{\top} H W_{e o} & =\Lambda_{H S} \Lambda_{S T} .
\end{aligned}
$$

We remark that $\Gamma_{o}$ and $\Gamma_{c}$ are degrees of freedom in the selection of $S$ and $T$, respectively. These matrices can be selected in order to improve the error bound or preserve more particular structures as is illustrated in Section 5.

\section{$5 \quad$ Examples}

In this section we present two examples to illustrate the applicability of the results reported in previous sections. Both examples represent physical systems, where, the first one is a mass-spring-damper mechanical system. While, the second example represents an RLC circuits network. 


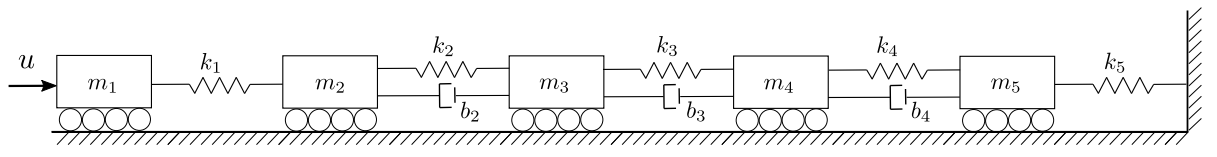

Figure 1: Mass-spring-damper network

\subsection{Mechanical system}

Consider five mass-spring-damper systems interconnected in series as shown in Fig. 1. The dynamics that describe this network of mechanical systems are given by

$$
\begin{aligned}
{\left[\begin{array}{c}
\dot{q} \\
\dot{p}
\end{array}\right]=} & \underbrace{\left[\begin{array}{cc}
\mathbf{0}_{5 \times 5} & I_{5} \\
-I_{5} & -R_{2}
\end{array}\right]}_{F} \underbrace{\left[\begin{array}{cc}
K & \mathbf{0}_{5 \times 5} \\
\mathbf{0}_{5 \times 5} & M^{-1}
\end{array}\right]}_{H}\left[\begin{array}{c}
q \\
p
\end{array}\right]+\underbrace{\left[\begin{array}{c}
\mathbf{0}_{5} \\
G
\end{array}\right]}_{B} u \\
G= & {\left[\begin{array}{c}
1 \\
\mathbf{0}_{4}
\end{array}\right], \quad M=\operatorname{diag}\left\{m_{1}, m_{2}, m_{3}, m_{4}, m_{5}\right\} } \\
K= & {\left[\begin{array}{ccccc}
k_{1} & -k_{1} & 0 & 0 & 0 \\
-k_{1} & k_{1}+k_{2} & -k_{2} & 0 & 0 \\
0 & -k_{2} & k_{2}+k_{3} & -k_{3} & 0 \\
0 & 0 & -k_{3} & k_{3}+k_{4} & -k_{4} \\
0 & 0 & 0 & -k_{4} & k_{4}+k_{5}
\end{array}\right], } \\
R_{2}= & {\left[\begin{array}{ccccc}
0 & 0 & 0 & 0 & 0 \\
0 & b_{2} & -b_{2} & 0 & 0 \\
0 & -b_{2} & b_{2}+b_{3} & -b_{3} & 0 \\
0 & 0 & -b_{3} & b_{3}+b_{4} & -b_{4} \\
0 & 0 & 0 & -b_{4} & b_{4}
\end{array}\right], }
\end{aligned}
$$

where $q, p \in \mathbb{R}^{5}$, which is in $\mathrm{PH}$ form.

The objective is to reduce the order of the model and ensure that the $\mathrm{PH}$ structure is preserved. Note that, independently of $\delta>0$, this system does not satisfy condition (65), and thus the Hamiltonian matrix cannot be proposed as a generalized Gramian. At this point, we remark that this system is neither controllable nor observable, but Assumption 1 holds.

Table 1:

Parameters of the mechanical system

\begin{tabular}{|l|l|}
\hline$b_{2}$ & $50[\mathrm{~kg} / \mathrm{s}]$ \\
\hline$b_{3}$ & $20[\mathrm{~kg} / \mathrm{s}]$ \\
\hline$b_{4}$ & $5[\mathrm{~kg} / \mathrm{s}]$ \\
\hline
\end{tabular}

\begin{tabular}{|l|l|}
\hline$m_{1}$ & $1.5[\mathrm{~kg}]$ \\
\hline$m_{2}$ & $0.5[\mathrm{~kg}]$ \\
\hline$m_{3}$ & $4[\mathrm{~kg}]$ \\
\hline$m_{4}$ & $2[\mathrm{~kg}]$ \\
\hline$m_{5}$ & $1.25[\mathrm{~kg}]$ \\
\hline
\end{tabular}

\begin{tabular}{|l|l|}
\hline$k_{1}$ & $4\left[\mathrm{~kg} / \mathrm{s}^{2}\right]$ \\
\hline$k_{2}$ & $7\left[\mathrm{~kg} / \mathrm{s}^{2}\right]$ \\
\hline$k_{3}$ & $2\left[\mathrm{~kg} / \mathrm{s}^{2}\right]$ \\
\hline$k_{4}$ & $5\left[\mathrm{~kg} / \mathrm{s}^{2}\right]$ \\
\hline$k_{5}$ & $3\left[\mathrm{~kg} / \mathrm{s}^{2}\right]$ \\
\hline
\end{tabular}

Based on the results presented in Section 4.2, we first adopt the generalized 
balanced truncation approach. To this end, we look for a solution $\breve{P}$ to the inequality (67) such that $X_{c}>0$. Hence, if the conditions established in Proposition 3 are satisfied, then we propose $W=W_{g c}$. In order to reduce the order of system (95) via generalized balanced truncation, we proceed as follows:

- We propose a positive definite matrix $\breve{X}_{c} \in \mathbb{R}^{n}$ to write the inequality (67) as an equality, that is,

$$
-B B^{\top}-\breve{X}_{c}=F H \breve{P}+\breve{P} H F^{\top}
$$

- We find $\breve{P}$ that solves (96).

- We look for a diagonal matrix that solves (69). If such diagonal matrix exists, then we propose $W=W_{g c}$, with $W_{g c}$ defined in (71). Notice that the singular values of the system are contained in $\Lambda_{Q P}$.

- We truncate the system and we obtain the reduced order model.

For illustration purposes, we consider the values given in Table 1, and we fix $\breve{X}_{c}=I_{10} \times 10^{-5}$. Hence, using Matlab, we find $\breve{P}$ that solves (96) which is given by (112), see the Appendix. Moreover, we use Matlab to solve the inequality (69), obtaining the solution

$$
\begin{aligned}
\Lambda_{Q P}= & \operatorname{diag}\{4.374,4.316,2.755,2.564, \\
& 1.188,0.626,0.482,0.324,0.155,0.070\} .
\end{aligned}
$$

Therefore, it follows from Proposition 3 that the reduced order model preserves the $\mathrm{PH}$ structure.

Now, for the sake of comparison we study the extended balancing case. Towards this end, we proceed as follows:

- We consider the matrices $\breve{X}_{c}$ and $\breve{P}$ used during the generalized balancing procedure.

- We propose $\Gamma_{c}$ and $\beta$ such that $T$, given in (25), solves (11).

- We look for a diagonal matrix $\Lambda_{S T}$ that solves (78). If such matrix exists, then we select $S$ as in (80), with $Q$ given in (79).

- We truncate the system and we obtain the reduced order model.

To illustrate the methodology, we replace $T=\left(\beta \breve{P}^{-1}+\Gamma_{c}\right)^{-1}$ in (11). Hence, using Matlab, we solve this equation for $\beta$ and a symmetric matrix $\Gamma_{c}$. As a result, we obtain

$$
\beta=4.8021 \times 10^{7}
$$

and the matrix given in (113), see the Appendix. Then, we fix $\alpha=\beta$ and we look for a solution $\Lambda_{Q T}$ to the inequality (78). Such a diagonal matrix is obtained by Matlab's LMI solver. Finally, we fix $\Lambda_{S T}=\frac{1}{\sqrt{\alpha}} \Lambda_{Q T}$ to obtain

$$
\begin{aligned}
\Lambda_{S T}= & \operatorname{diag}\{3.71,3.666,2.415,2.218,0.976, \\
& 0.543,0.401,0.245,0.099,0.041\} .
\end{aligned}
$$

Thus, it follows from Proposition 5 that the reduced order model preserves the PH structure. In this example, we chose $\Gamma_{c}$ based on the value of the four smallest entries of $\Lambda_{S T}$. We tuned $\Gamma_{c}$ by trial and error for illustration purposes. However, to improve the results, this matrix can be computed by solving an optimization problem.

In order to compare the error bounds of both balancing approaches, we truncate 
four states of the original system, that is $k=6$. Accordingly, for the generalized balancing case we get

$$
\left\|\Sigma-\Sigma_{r}\right\|_{\infty} \leq 2.06,
$$

and for extended balancing we have

$$
\left\|\Sigma-\Sigma_{r}\right\|_{\infty} \leq 1.57
$$

To compare the behavior of system (95) and both reduced order systems, obtained via generalized balanced truncation and extended balanced truncation, we perform simulations under initial conditions $\bar{x}=\mathbf{0}_{10}$ for the balanced system, $\hat{x}=\mathbf{0}_{6}$ for both reduced order systems, and the input signal depicted in Fig. 2. Figure 3 shows the comparison between the outputs of the balanced system and the reduced order system obtained via generalized balanced truncation. Anal-

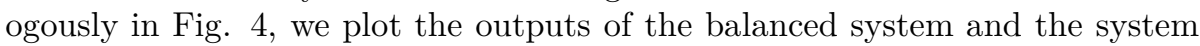
obtained through extended balanced truncation. From Figs. 3 and 4 we notice that the output of the balanced system, plotted in black, and the outputs of the reduced order systems, depicted in red, significantly similar. Thus we conclude that, with both balanced truncation approaches, we preserve the PH structure of the original system, and the response of the reduced order systems to a given input is similar to the response of the original system.

We remark that while the $\mathrm{PH}$ structure is preserved for generalized and extended balanced truncation, the comparison between (99) and (100) shows that the error bound obtained from the latter balancing method is smaller. However, in the simulations we performed, for the real error, the smaller error bound did not make much difference. This situation can be observed in Fig. [5, where we present in black the difference between the output of the balanced system and the output of the reduced order system obtained through generalized balanced truncation, and in red we plot the difference between the output of the balanced system and the output of the reduced order system obtained through extended balanced truncation.

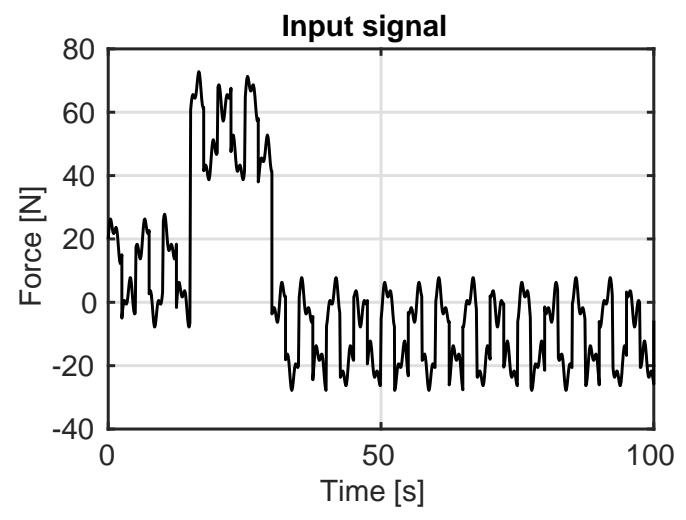

Figure 2: Signal $u$. 


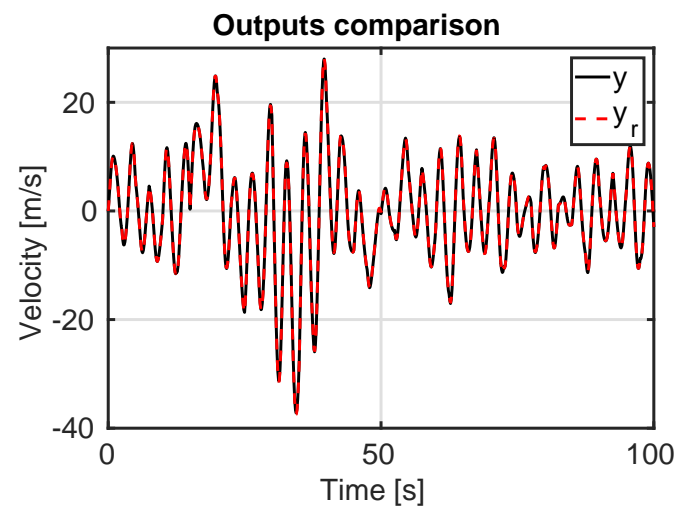

Figure 3: Outputs of the balanced system and the reduced order system obtained via generalized balanced truncation.

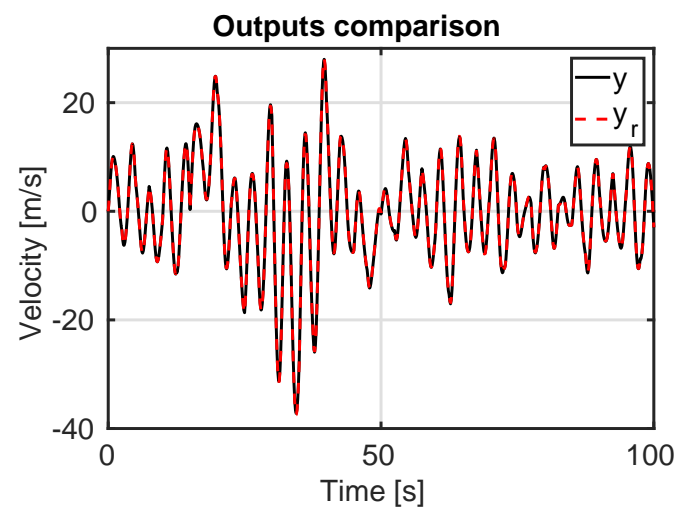

Figure 4: Outputs of the balanced system and the reduced order system obtained via generalized balanced truncation.

\subsection{RLC circuit}

Consider the RLC network depicted in Fig. 6 which admits a PH representation of the form (59) with

$$
\begin{aligned}
J= & {\left[\begin{array}{cc}
\mathbf{0}_{5 \times 5} & J_{1} \\
-J_{1}^{\top} & \mathbf{0}_{5 \times 5}
\end{array}\right], \quad R=\left[\begin{array}{cc}
R_{C}^{-1} & \mathbf{0}_{5 \times 5} \\
\mathbf{0}_{5 \times 5} & R_{L}
\end{array}\right], } \\
H= & \operatorname{diag}\left\{\frac{1}{C_{1}}, \frac{1}{C_{2}}, \frac{1}{C_{3}}, \frac{1}{C_{4}}, \frac{1}{C_{5}}, \frac{1}{L_{1}}, \frac{1}{L_{2}}, \frac{1}{L_{3}}, \frac{1}{L_{4}}, \frac{1}{L_{5}}\right\}, \\
R_{C}= & \operatorname{diag}\left\{R_{C_{1}}, R_{C_{2}}, R_{C_{3}}, R_{C_{4}}, R_{C_{5}}\right\}, \\
R_{L}= & \operatorname{diag}\left\{R_{L_{1}}, R_{L_{2}}, R_{L_{3}}, R_{L_{4}}, R_{L_{5}}\right\}, \\
J_{1}= & {\left[\begin{array}{ccccc}
1 & -1 & 0 & 0 & 0 \\
0 & 1 & -1 & 0 & 0 \\
0 & 0 & 1 & -1 & 0 \\
0 & 0 & 0 & 1 & -1 \\
0 & 0 & 0 & 0 & 1
\end{array}\right], B=\left[\begin{array}{c}
\mathbf{0}_{5} \\
1 \\
\mathbf{0} 4
\end{array}\right], }
\end{aligned}
$$




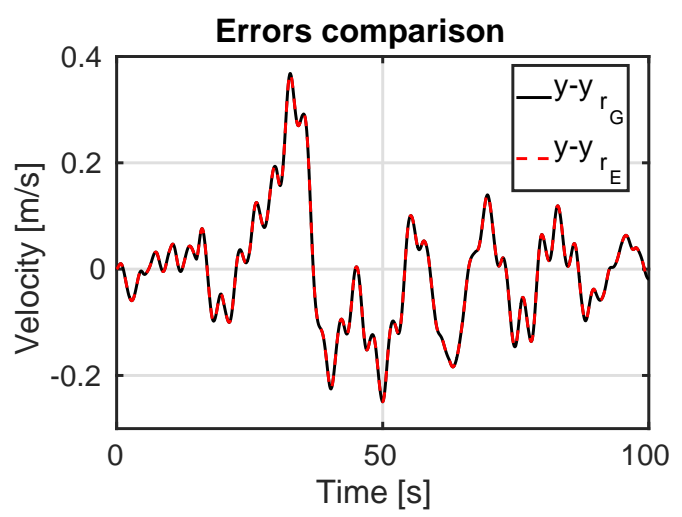

Figure 5: Error between the outputs of the balanced system and the reduced order systems.

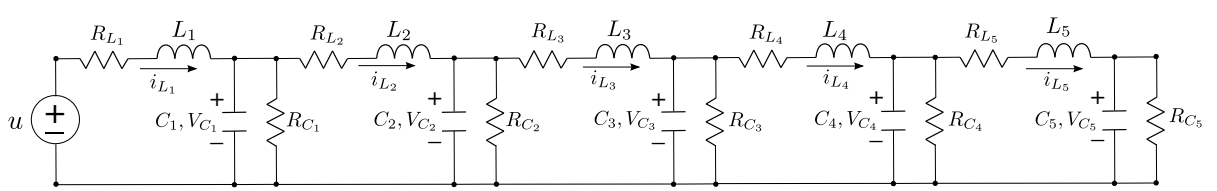

Figure 6: RLC network

where $x_{i}$ are the charges in the capacitors and $x_{5+i}$ denote the fluxes in the inductors, for $i=1, \cdots, 5$.

The objective is to reduce the order of the model and obtain a PH system that has a physical interpretation as an RLC circuit. Accordingly, we require that the reduced PH system has a diagonal damping matrix, and the interconnection matrix must be skew-symmetric and block anti-diagonal, which is more particular than the standard PH structure given in (59). We stress the fact that the matrices $J, R$, and $H$ can be decomposed in block matrices whose dimension depend on the number of inductors and capacitors, in this case 5. Moreover, $H$ is already diagonal. Thus, a block diagonal transformation $2 \mathrm{~W}$ ensures that $\bar{H}$ remains diagonal, and the block structure that determines the RLC architecture of the system is not affected.

Note that the damping matrix $R$ has full rank. Hence, we can select

$$
Q=\delta_{o} H, \quad \breve{P}=\delta_{c} H^{-1},
$$

where $\delta_{o}$ and $\delta_{c}$ are positive constants such that (65) holds. Therefore, both generalized Gramians are diagonal and the resulting transformation $W_{g}$ will not modify the structure of the original system. Nevertheless, in such case, the Hankel singular values are given by

$$
\Lambda_{Q P}=\sqrt{\delta_{o} \delta_{c}} I_{n} .
$$

\footnotetext{
${ }^{2}$ Where the dimension of the blocks is again related to the number of capacitors and inductors.
} 
Table 2:

Parameters of the RLC network

\begin{tabular}{|l|c|}
\hline$R_{C_{1}}$ & $270[\Omega]$ \\
\hline$R_{C_{2}}$ & $1[k \Omega]$ \\
\hline$R_{C_{3}}$ & $330[\Omega]$ \\
\hline$R_{C_{4}}$ & $1.5[k \Omega]$ \\
\hline$R_{C_{5}}$ & $220[\Omega]$ \\
\hline$R_{L_{1}}$ & $4.7[\Omega]$ \\
\hline$R_{L_{2}}$ & $3.9[\Omega]$ \\
\hline$R_{L_{3}}$ & $2.2[\Omega]$ \\
\hline$R_{L_{4}}$ & $2.74[\Omega]$ \\
\hline$R_{L_{5}}$ & $3.92[\Omega]$ \\
\hline
\end{tabular}

\begin{tabular}{|l|c|}
\hline$C_{1}$ & $2.2[\mathrm{mF}]$ \\
\hline$C_{2}$ & $1[\mathrm{mF}]$ \\
\hline$C_{3}$ & $3.3[\mathrm{mF}]$ \\
\hline$C_{4}$ & $15[\mu \mathrm{F}]$ \\
\hline$C_{5}$ & $4.7[\mu \mathrm{F}]$ \\
\hline$L_{1}$ & $10[\mathrm{mH}]$ \\
\hline$L_{2}$ & $4.3[\mathrm{mH}]$ \\
\hline$L_{3}$ & $2.7[\mathrm{mH}]$ \\
\hline$L_{4}$ & $6.2[\mu \mathrm{H}]$ \\
\hline$L_{5}$ & $3[\mu \mathrm{H}]$ \\
\hline
\end{tabular}

Since all the entries the matrix $\Lambda_{Q P}$ are equal, the criterion of truncating the states related to the smallest singular values is impractical and further information is required to decide which states can be removed. To deal with this situation, we proceed as follows:

- We fix $\breve{P}$ as in (102).

- We propose $\beta$ and a diagonal matrix $\Gamma_{c}$ sucht that $T$, defined in (25), solves the LMI (11).

- We look for a matrix $\Lambda_{Q T}$ that solves the inequality (78). We stress the fact that, in this case $Q$, given by (79), is a diagonal matrix.

- We fix $\alpha=\beta$ and we look for a diagonal matrix $\Gamma_{o}$ such that $S$, given by (15), solves the LMI (10).

- We find a transformation that balances the system. Then, we truncate the system to obtain the reduced order model.

To illustrate the methodology, we consider the values in Table 2 . Then, we propose ${ }^{3} \delta_{c}=0.11$ in (102). Hence, the design parameters

$$
\begin{aligned}
\Gamma_{c} & =-\operatorname{diag}\{14,4.9,3.7,0,0,190,600,350,3.9,10\} \\
\beta & =\alpha=5 \times 10^{8}
\end{aligned}
$$

ensure that

$$
\begin{aligned}
T= & \operatorname{diag}\{0.08,0.18,0.06,121.21,38.68, \\
& 0.02,0.04,0.07,29.66,64.52\} \times 10^{-4},
\end{aligned}
$$

solves (11). Now, using Matlab, we solve the inequality (78) to obtain

$$
\begin{aligned}
\Lambda_{Q T}= & \operatorname{diag}\{5.89,5.85,6.23,6.56,6.83 \\
& 6.93,6.5,6.63,5.84,5.61\} \times 10^{3} \\
Q= & \operatorname{diag}\{0.39,0.78,0.21,414.89,134.25 \\
& 0.09,0.19,0.28,101.3,202.93\} \times 10^{3}
\end{aligned}
$$

Moreover, we propose

$$
\Gamma_{o}=\operatorname{diag}\{0,0,0,0.2,0.1,0,0,0,1,5\} \times 10^{12} .
$$

\footnotetext{
${ }^{3} \mathrm{~A}$ large $\delta_{c}$ is translated in large values of the entries of $\breve{P}$ which can, potentially, produce large singular values.
} 
Hence, we replace $Q$, given in (105), and (106) in (15). Accordingly,

$$
\begin{aligned}
S= & \operatorname{diag}\{0.08,0.16,0.04,82.9,26.81, \\
& 0.02,0.04,0.06,19.87,38.68\} \times 10^{-5},
\end{aligned}
$$

which solves the LMI (10).

Note that

$$
H T^{-1} S=S T^{-1} H
$$

Hence, it follows from Theorem 4 that there exist a transformation $W$ that balances the system and preserve the $\mathrm{PH}$ structure. Moreover, the matrices $H, T$ and $S$ are diagonal. As a result, $W$ is a block diagonal matrix, thus, we can express the matrices $W$ and $\Lambda_{S T}$ as follows

$$
\begin{aligned}
W & =\operatorname{diag}\left\{W_{1}, W_{2}\right\} \\
\Lambda_{S T} & =\operatorname{diag}\left\{\Lambda_{S T_{1}}, \Lambda_{S T_{2}}\right\} \\
\Lambda_{S T_{i}} & =\operatorname{diag}\left\{\sigma_{i_{1}}, \cdots, \sigma_{i_{5}}\right\}, i=1,2,
\end{aligned}
$$

where

$$
\begin{aligned}
W_{1} & =\left[\begin{array}{ccccc}
629.3 & 0 & 0 & 0 & 0 \\
0 & 433 & 0 & 0 & 0 \\
0 & 0 & 807.2 & 0 & 0 \\
0 & 0 & 0 & 0 & 17.8 \\
0 & 0 & 0 & 31.3 & 0
\end{array}\right] \\
W_{2} & =\operatorname{diag}\{1332,892.1,714.2,36.1,25.2\} \\
\Lambda_{S T_{1}} & =\operatorname{diag}\{0.31,0.29,0.28,0.26,0.26\} \\
\Lambda_{S T_{2}} & =\operatorname{diag}\{0.31,0.3,0.29,0.26,0.24\} .
\end{aligned}
$$

The criterion to choose the parameters $\Gamma_{c}, \Gamma_{o}$, and $\beta$ differs from the example studied in Section 5.1. In this case, we want to have a significant contrast in the entries of $\Lambda_{S T}$ to have information about which states can be truncated without affecting the response of the reduced-order system significantly. The mentioned parameters were selected by trial and error for the sake of illustration, but these might be computed by solving an optimization problem.

At this point, we make three observations regarding the preservation of the RLC structure:

(i) As mentioned before, to preserve the RLC structure it is necessary to ensure that $W$ is a block diagonal matrix.

(ii) We are truncating the states related to the entries of $\Lambda_{S T}$ in pairs, that is, one state related to one element of $\Lambda_{S T_{1}}$ and one state related to one entry from $\Lambda_{S T_{2}}$. The physical interpretation of this condition is that we are removing the same number of inductors and capacitors.

(iii) By fixing $\Gamma_{o}$ and $\Gamma_{c}$ different from zero, we ensure that the entries of $\Lambda_{S T}$ are different. Then, we can apply the criterion of truncating the states related to the smallest entries of each submatrix $\Lambda_{S T_{i}}$.

For illustration purposes, we truncate the states related to $\sigma_{i_{4}}, \sigma_{i_{5}}$. In such a 


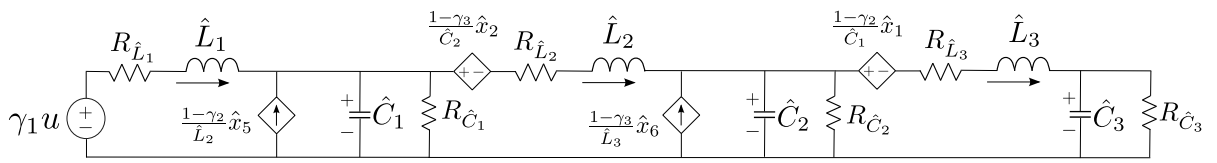

Figure 7: Reduced RLC network

case, the reduced order model admits a $\mathrm{PH}$ representation with

$$
\begin{aligned}
R_{r} & =\operatorname{diag}\left\{R_{C_{r}}^{-1}, R_{L_{r}}\right\}, \quad J_{r}=\left[\begin{array}{cc}
\mathbf{0}_{3 \times 3} & J_{1_{r}} \\
J_{1_{r}}^{\top} & \mathbf{0}_{3 \times 3}
\end{array}\right], \\
H_{r} & =\operatorname{diag}\left\{\frac{1}{C_{1_{r}}}, \frac{1}{C_{2_{r}}}, \frac{1}{C_{3_{r}}}, \frac{1}{L_{1_{r}}}, \frac{1}{L_{2_{r}}}, \frac{1}{L_{3_{r}}}\right\}, \\
R_{C_{r}} & :=\operatorname{diag}\left\{R_{C_{1_{r}}}, R_{C_{2_{r}}}, R_{C_{3_{r}}}\right\}, \\
R_{L_{r}} & :=\operatorname{diag}\left\{R_{L_{1_{r}}}, R_{L_{2_{r}}}, R_{L_{3_{r}}}\right\} \\
J_{1_{r}} & =\left[\begin{array}{ccc}
1 & -\gamma_{2} & 0 \\
0 & 1 & -\gamma_{3} \\
0 & 0 & 1
\end{array}\right], \quad B_{r}=\left[\begin{array}{l}
\mathbf{0}_{3} \\
\gamma_{1} \\
\mathbf{0}_{2}
\end{array}\right]
\end{aligned}
$$

and the values given in Table 3 . The error bound is given by

$$
\left\|\Sigma-\Sigma_{r}\right\|_{\infty} \leq 2.06,
$$

and the reduced order model admits the RLC realization depicted in Fig. 7 , where the states $\hat{x}_{i}$ represent the charges in the capacitors and $\hat{x}_{i+3}$ denote the fluxes in the inductors for $i=1,2,3$.

Table 3:

Parameters of the reduced RLC circuit

\begin{tabular}{|l|l|}
\hline$\gamma_{1}$ & $0.69 \times 10^{-4}$ \\
\hline$\gamma_{2}$ & 1.01 \\
\hline$\gamma_{3}$ & 1.53 \\
\hline
\end{tabular}

\begin{tabular}{|l|l|}
\hline$R_{C_{1_{r}}}$ & 127.55 \\
\hline$R_{C_{2 r}}$ & 485.34 \\
\hline$R_{C_{3 r}}$ & 373.01 \\
\hline$R_{L_{1_{r}}}$ & 2.22 \\
\hline$R_{L_{2 r}}$ & 1.89 \\
\hline$R_{L_{3 r}}$ & 2.49 \\
\hline
\end{tabular}

\begin{tabular}{|l|l|}
\hline$C_{1_{r}}$ & $4.66 \times 10^{-3}$ \\
\hline$C_{2_{r}}$ & $2.06 \times 10^{-3}$ \\
\hline$C_{3_{r}}$ & $2.92 \times 10^{-3}$ \\
\hline$L_{1_{r}}$ & $4.72 \times 10^{-3}$ \\
\hline$L_{2_{r}}$ & $2.09 \times 10^{-3}$ \\
\hline$L_{3_{r}}$ & $3.05 \times 10^{-3}$ \\
\hline
\end{tabular}

\section{Simulation results}

We carry out simulations to compare the behavior of the original system with:

- A reduced order system obtained via generalized balancing, where the generalized Gramians are chosen as in (102) and $\delta_{o}=\delta_{c}$.

- The reduced order system obtained through extended balanced truncation, with Gramians (104) and (107).

A first set of simulations is performed considering that the systems start at rest and the input signal depicted in Fig. 8, Figure 9 shows the outputs of the systems, where $y$ is the output of the balanced system, $y_{G}$ represents the 


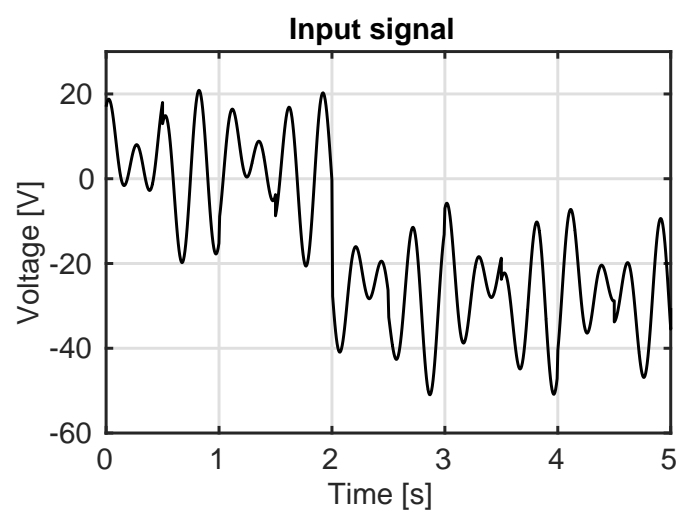

Figure 8: Input signal $u$.

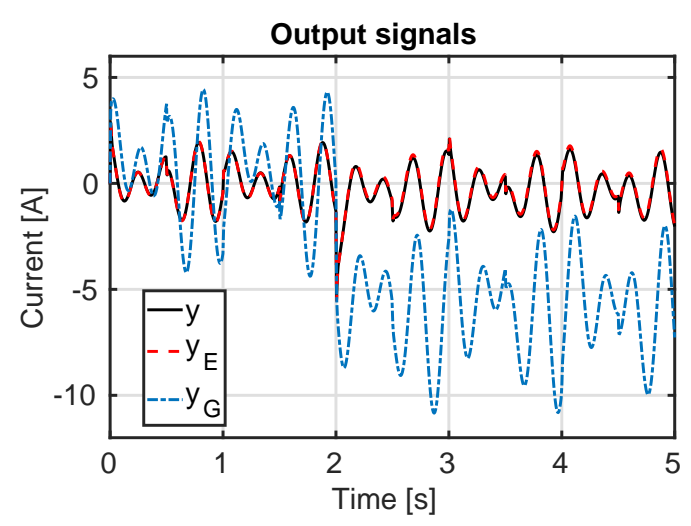

Figure 9: Plot of the different outputs.

output of the system obtained via generalized balancing, and $y_{E}$ corresponds to the output of the system obtained via extended balanced truncation. In Fig. 9 we can observe that the difference between $y_{E}$ and $y$ is rather small, while the output $y_{G}$ is considerably different from the output of the balanced system, this can be the result of truncating states without any justification in the generalized balanced truncation case. Figure 10 shows the plot of the difference $y-y_{G}$ and Fig. 11 depicts the difference $y-y_{E}$, if we compare both plots, we corroborate that - note that the scales of the plots are different- the error $y-y_{G}$ is noticeably bigger than the error $y-y_{E}$.

A second set of simulations is carried out considering the input shown in Fig. 12 and the systems starting at rest. Figure [13] shows the outputs of the systems, where it is clear that the output $y_{G}$, plotted in blue, is totally different from the output of the balanced system. As we discussed above, the reason for this difference is the lack of a criterion to truncate the states in the generalized balancing approach. On the other hand, we observe in Fig. 13 that the plot of $y_{E}$ approximates the behavior of $y$. Hence we conclude that, in this example, the matrices $\Gamma_{c}$ and $\Gamma_{o}$ can be exploited to reduce the error bound while preserving the physical interpretation of the original system. 


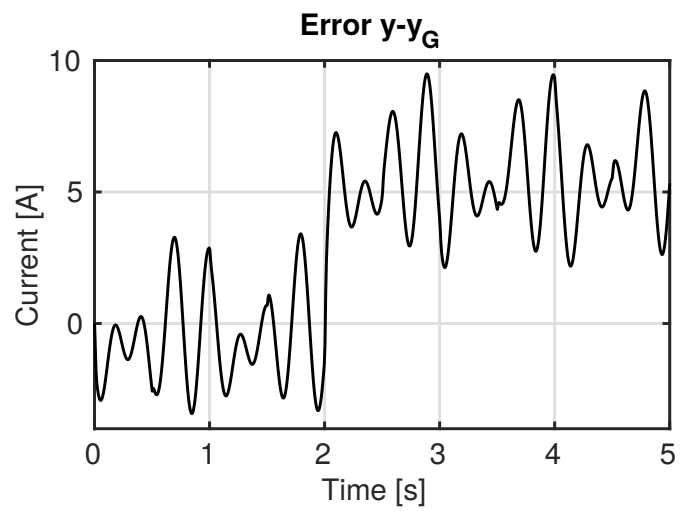

Figure 10: Plot of the error $y-y_{G}$.

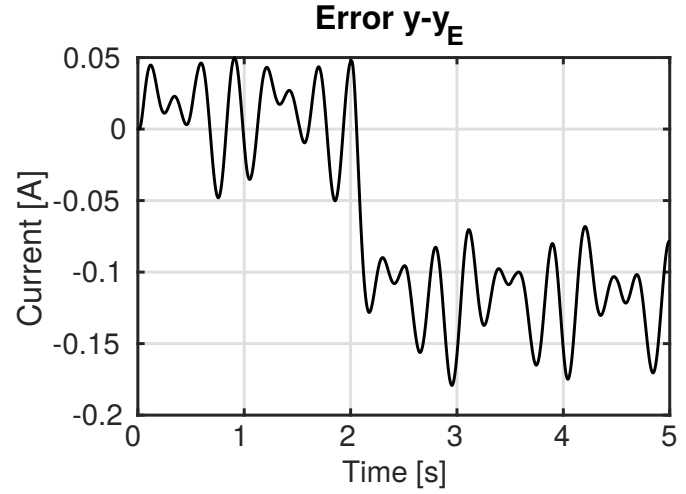

Figure 11: Plot of the error $y-y_{E}$.

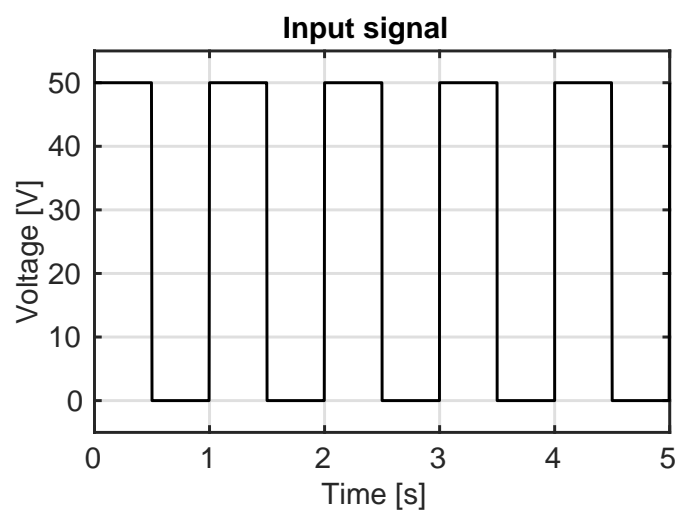

Figure 12: Input signal $u$. 


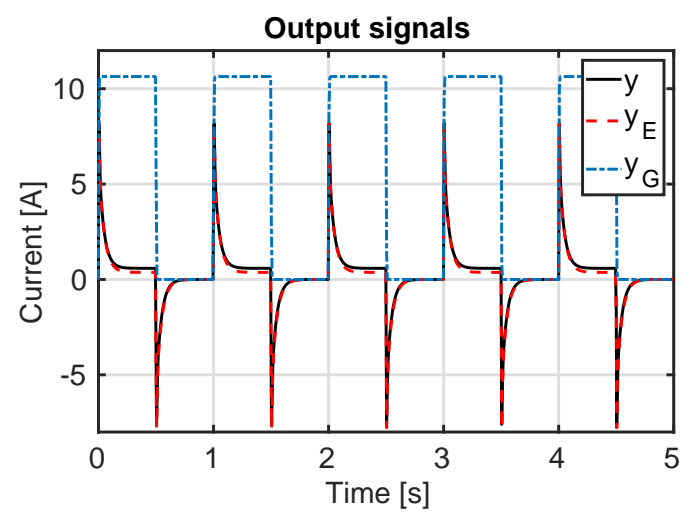

Figure 13: Plot of the different outputs.

\section{Concluding remarks}

In this paper we have provided sufficient conditions to ensure the existence of extended Gramians that are suitable to compute an error bound. Additionally, we have formulated an approach to preserve the $\mathrm{PH}$ structure for the truncated system by using generalized and extended Gramians. Furthermore, we have shown that the extended balancing is a versatile tool that can be used to obtain a smaller error bound or to preserve some particular structures, such as, an RLC structure.

The matrices related to the mechanical example are listed below

$$
\breve{P}=\left[\begin{array}{ccccc}
0.97 & 0.37 & 0.35 & 0.29 & 0.15 \\
0.37 & 0.46 & 0.44 & 0.39 & 0.26 \\
0.35 & 0.44 & 0.43 & 0.38 & 0.26 \\
0.29 & 0.39 & 0.38 & 0.35 & 0.24 \\
0.15 & 0.26 & 0.26 & 0.24 & 0.18 \\
0 & -0.13 & -0.06 & 0.03 & 0.08 \\
0.04 & 0 & 0 & 0 & 0 \\
0.15 & 0.03 & 0 & 0.04 & 0.06 \\
-0.05 & -0.01 & -0.02 & 0 & 0.02 \\
-0.07 & -0.01 & -0.02 & -0.01 & 0
\end{array}\right.
$$

$\left.\begin{array}{ccccc}0 & 0.04 & 0.15 & -0.05 & -0.07 \\ -0.13 & 0 & 0.03 & -0.01 & -0.01 \\ -0.06 & 0 & 0 & -0.02 & -0.02 \\ 0.03 & 0 & 0.04 & 0 & -0.01 \\ 0.08 & 0 & 0.06 & 0.02 & 0 \\ 3.77 & -0.19 & -1.56 & -0.78 & -0.55 \\ -0.19 & 0.04 & 0.32 & 0.15 & 0.08 \\ -1.56 & 0.32 & 2.52 & 1.19 & 0.63 \\ -0.78 & 0.15 & 1.19 & 0.58 & 0.32 \\ -0.55 & 0.08 & 0.63 & 0.32 & 0.18\end{array}\right]$




$$
\Gamma_{c}=\left[\begin{array}{ccccc}
0.05 & -0.1 & -0.07 & -0.05 & -0.03 \\
-0.1 & 0.01 & 0 & -0.01 & 0 \\
-0.07 & 0 & -0.01 & -0.02 & -0.01 \\
-0.05 & -0.01 & -0.02 & -0.02 & -0.01 \\
-0.03 & 0 & -0.01 & -0.01 & -0.01 \\
1.63 & -0.56 & -0.57 & -0.54 & -0.5 \\
-0.12 & 0.03 & 0.04 & 0.04 & 0.04 \\
-1.01 & 0.31 & 0.33 & 0.35 & 0.34 \\
-0.51 & 0.17 & 0.17 & 0.18 & 0.17 \\
-0.32 & 0.11 & 0.11 & 0.11 & 0.1 \\
1.63 & -0.12 & -1.01 & -0.51 & -0.32 \\
-0.56 & 0.03 & 0.31 & 0.17 & 0.11 \\
-0.57 & 0.04 & 0.33 & 0.17 & 0.11 \\
-0.54 & 0.04 & 0.35 & 0.18 & 0.11 \\
-0.5 & 0.04 & 0.34 & 0.17 & 0.1 \\
-0.29 & 0.18 & 0.8 & 0.11 & -0.02 \\
0.18 & -0.02 & -0.11 & -0.04 & -0.02 \\
0.8 & -0.11 & -0.52 & -0.09 & -0.03 \\
0.11 & -0.04 & -0.09 & 0.04 & 0.04 \\
-0.02 & -0.02 & -0.03 & 0.04 & 0.04
\end{array}\right]
$$

\section{References}

[1] Athanasios C. Antoulas. Approximation of large-scale dynamical systems. Siam, Philadelphia, 2005

[2] TK Caughey. Classical normal modes in damped linear dynamic systems. Journal of Applied Mechanics, 27(2):269-271, 1960.

[3] Xiaodong Cheng, Jacquelien MA Scherpen, and Bart Besselink. Balanced truncation of networked linear passive systems. Automatica, 104:17-25, 2019.

[4] M.C. de Oliveira, J. Bernussou, and J.C. Geromel. A new discrete-time robust stability condition. Systems \& Control Letters, 37:261-265, 1999.

[5] M.C. de Oliveira, J.C. Geromel, and J. Bernoussou. Extended $\mathcal{H}_{2}$ and $\mathcal{H}_{\infty}$ norm characterizations and controller parameterizations for discrete-time systems. Int. J. Control, 75(9):666679, 2002.

[6] V. Duindam, A. Macchelli, S. Stramigioli, and H. Bruyninckx. Modeling and control of complex physical systems: the port-Hamiltonian approach. Springer Science \& Business Media, 2009.

[7] Geir E Dullerud and Fernando Paganini. A course in robust control theory: a convex approach, volume 36. Springer Science \& Business Media, 2013.

[8] Kenji Fujimoto. Balanced realization and model order reduction for port-hamiltonian systems. Journal of System Design and Dynamics, 2(3):694-702, 2008.

[9] Kenji Fujimoto and Jacquelien M.A. Scherpen. Balanced realization and model order reduction for nonlinear systems based on singular value analysis. SIAM Journal on Control and Optimization, 48(7):4591-4623, 2010.

[10] Keith Glover. All optimal Hankel-norm approximations of linear multivariable systems and their $\mathcal{L}_{\infty}$-error bounds. International journal of control, 39(6):1115-1193, 1984.

[11] D Hinrichsen and AJ Pritchard. An improved error estimate for reduced-order models of discrete-time systems. IEEE Transactions on Automatic Control, 35(3):317-320, 1990.

[12] R. Horn and C. Johnson. Matrix Analysis. Cambridge University Press, 1985

[13] Yu Kawano and Jacquelien MA Scherpen. Structure preserving truncation of nonlinear port hamiltonian systems. IEEE Transactions on Automatic Control, 2018.

[14] Bruce C. Moore. Principal component analysis in linear systems: Controllability, observability, and model reduction. IEEE transactions on automatic control, 26(1):17-32, 1981.

[15] Mikhail Alekseevich Novikov. Simultaneous diagonalization of three real symmetric matrices. Russian Mathematics, 58(12):59-69, 2014.

[16] Henrik Sandberg. Model reduction of linear systems using extended balanced truncation. In 2008 American Control Conference, pages 4654-4659. IEEE, 2008.

[17] Henrik Sandberg. An extension to balanced truncation with application to structured model reduction. IEEE Transactions on Automatic Control, 55(4):1038-1043, 2010.

[18] Jacquelien M.A. Scherpen. The control handbook: control system advanced methods, chapter Balanced realizations, model order reduction, and the Hankel operator, pages 1-24. Taylor \& Francis group, second edition, 2011.

[19] Jacquelien M.A. Scherpen and Kenji Fujimoto. Extended balanced truncation for continuous time LTI systems. In Control Conference (ECC), 2018 European. IEEE, 2018.

[20] A. J. van der Schaft. $L_{2}$-Gain and Passivity techniques in nonlinear control. Springer, Berlin, third edition, 2016.

[21] Jan C. Willems. Model reduction by balancing. Lecture retrieved from http://homes.esat.kuleuven.be/7Ejwillems/Lectures/2002 /modelred.pdf, 2002. 
[22] Kemin Zhou, John C. Doyle, and Keith Glover. Robust and optimal control. Prentice-Hall, New Jersey, 1996. 